\title{
Strategic Behavior and Social Outcomes in a Bottleneck Queue: Experimental Evidence
}

\author{
by \\ Jesper Breinbjerg, \\ Alexander Sebald \\ and \\ Lars Peter Østerdal
}

Discussion Papers on Business and Economics

No. $12 / 2014$

FURTHER INFORMATION

Department of Business and Economics Faculty of Business and Social Sciences University of Southern Denmark Campusvej 55 DK-5230 Odense M Denmark

Tel.: +45 65503271

Fax: +4565503237

E-mail: lho@sam.sdu.dk http://www.sdu.dk/ivoe 


\title{
Strategic Behavior and Social Outcomes in a Bottleneck Queue: Experimental Evidence*
}

\author{
Jesper Breinbjerg ${ }^{\dagger} \quad$ Alexander Sebald ${ }^{\ddagger} \quad$ Lars Peter $\varnothing_{\text {sterdal }}{ }^{\S}$
}

July 25, 2014

\begin{abstract}
We consider a class of three-player queuing games where players independently choose when to arrive at a bottleneck facility that serves only one at a time. Players are impatient for service but cannot arrive before the facility opens and they dislike time spent in queue. We derive the equilibrium arrivals under the first-in-first-out (FIFO), last-in-first-out (LIFO), and service-in-random-order (SIRO) queue disciplines and compare these equilibrium predictions to outcomes from a laboratory experiment. LIFO provides higher equilibrium welfare than FIFO and SIRO since the players arrive such that lower congestion is induced. Experimental evidence confirms that employing different queue disciplines indeed affects the strategic behavior of players and thereby the level of congestion. The experimental participants do not, however, behave as prescribed by the equilibrium predictions. They obtain significantly higher welfare than prescribed by equilibrium under all queue disciplines. Our results moreover suggest that people perceive LIFO as the most unfair of the three disciplines although the theoretical results suggest that it is welfare optimal.
\end{abstract}

Keywords: Queue disciplines; congestion; equilibrium; experiments; fairness.

JEL-Classifications: C72, D62, D63, R41.

${ }^{*}$ We thank Refael Hassin, Martin Osborne, and conference and seminar participants at Copenhagen, Odense, Oxford, Toronto, Boston, and Kraków for helpful comments. The financial support from the Danish Council for Strategic Research and the The Danish Council for Independent Research | Social Sciences (Grant IDs: DFF-1327-00097 and DFF-10-081368) is gratefully acknowledged. Lastly, a special thanks to the Department of Economics of the University of Toronto for the warm hospitality provided during Jesper Breinbjerg's research visit.

${ }^{\dagger}$ Department of Business and Economics, University of Southern Denmark, Campusvej 55, DK-5230 Odense M. Email: jnb@sam.sdu.dk.

${ }^{\ddagger}$ Department of Economics, University of Copenhagen, Øster Farimagsgade 5, Building 26, DK-1353, Copenhagen K. Email: alexander.sebald@econ.ku.dk.

${ }^{\S}$ Department of Business and Economics, University of Southern Denmark, Campusvej 55, DK-5230 Odense M. Email: Ipro@sam.sdu.dk. 


\section{Introduction}

In many everyday situations, we spend time in a queue waiting to be served. Examples range from small-scale situations, such as patients showing up at a walk-in clinic after opening time where pre-booking appointments is not possible, to large-scale situations, such as American citizens applying for ObamaCare (the Affordable Care Act) through the federal online marketplace. The inefficiencies brought about by such bottlenecks where agents fail to coordinate their time of arrivals often create congestion and therefore time spent queuing.

This paper considers a discrete queuing game, where players choose when to arrive for service after the opening of a bottleneck facility. The players want to be served as early as possible and spend minimal time in the queue. With limited service capacity at the bottleneck, the optimal arrival strategy depends on the arrival of the other players since congestion causes service delays and therefore longer queuing time. The strategic behavior within queuing games is often analyzed by their resulting equilibrium arrival pattern. However, from the perspective of social welfare maximization, these equilibrium arrivals may not provide the welfare optimal solution (viz., no queuing time) since players seek to maximize their own utility without regard for the others' welfare (see Hassin and Haviv, 2003 for an excellent review of a sizeable part of this literature). Thus, social planners should take such behavior into consideration and design queuing systems such that their procedural characteristics induce optimal strategic behavior.

The existing literature has mostly focused on the first-in-first-out (FIFO) queue discipline in games with strategic arrivals (e.g., Vickrey, 1969; Glazer and Hassin, 1983; Arnott et al., 1999; Hassin and Kleiner, 2011 and references therein). However, while FIFO is intuitively fair and acceptable to most players, it may not be the optimal way of settling a queue (Hassin, 1985). As queue disciplines govern the service priority of arrived players, they influence the strategic incentives for arrival. In recent years, the role of the queue discipline has received increasing attention in the literature. de Palma and Fosgerau (2013) consider a bottleneck model with a continuum of risk-averse agents and show that all stochastic disciplines that give priority to early arrivals (to a vanishing degree) provide the same equilibrium welfare. Platz and Østerdal (2012) study a class of queuing games with a continuum of players and no possibility of queuing before opening time, and show that the FIFO discipline induces the worst Nash equilibrium in terms of equilibrium welfare while the last-in-first-out (LIFO) queue discipline induces the best.

Motivated by these findings, we theoretically and experimentally analyze the strategic behavior of players, social welfare, and the players' fairness perceptions in a discrete queuing game using three different queue disciplines. Specifically, to create a theoretical benchmark for our experimental analysis, we first derive pure and (symmetric) mixed strategy Nash equilibrium arrivals in a three-player, discrete-time queuing game under three well-known queue disciplines: FIFO, LIFO, and service-in-random-order (SIRO), and compare the quantitative differences between the corresponding equilibrium welfare 
levels. In our theoretical analysis we establish results for general values of the model parameters. Subsequently, we experimentally test our behavioral predictions and welfare properties for specific parameter values. We restrict our attention to three-player queuing games to enforce computational simplicity in establishing the general equilibrium arrivals, and to obtain more experimental game repetitions for a given pool of participants.

A first glance at the results: First, our experimental analysis shows that queue disciplines affect strategic behavior differently. That is, the arrival patterns differ substantially between the queue disciplines. Secondly, independent of the queue discipline, subjects largely do not behave in accordance with what is predicted by our theoretical equilibrium analysis. For all queue disciplines, the arrival distributions differ significantly from the predicted equilibrium distributions. Third, despite the fact that LIFO theoretically provides the highest welfare given the specific parameters used in the experiment, the welfare provided by LIFO in our experiment is not significantly different from that provided by FIFO and SIRO. Lastly, notwithstanding deviations from individual optimizing behavior, across all queue disciplines subjects' expected payoffs are higher in our experiment compared to our theoretical equilibrium predictions. The reason why subjects successfully outperform the equilibrium in regards to expected payoff is that they reduced the expected queuing time by leveling out their arrivals. Compared to the equilibrium predictions, subjects arrived later and thereby reduced congestion.

The paper is organized as follows: Section 2 presents related literature. Section 3 introduces the queuing game and model assumptions. Section 4 establishes and compares the equilibrium arrivals under each queue discipline. Section 5 introduces the experimental design and hypothesis for testing. Section 6 presents the experimental results. Section 7 interprets and discusses the results. The supplement contains proofs and other technical material.

\section{Related Literature}

Queuing problems have been analyzed by economists and operations researchers for decades and explored for various types of queuing systems (see e.g. Hall, 1991, and Hillier, 1990, for surveys on operations research literature and Small and Verhoef, 2007 for urban transportation literature).

Our model setup is related to the classical bottleneck model of Vickrey (1969) that models congestion arising at a bottleneck in the context of morning commute and trip timing. The model assumes that a single bottleneck, open at all times, serves a non-atomic set of commuters. Delay occurs when the traffic flow exceeds the capacity of the bottleneck. Each commuter has a preferred time to pass the bottleneck. There is a cost for arriving earlier than desired, and a cost for a late arrival. The original model was further analyzed and extended by Arnott et al. (1993), Ostubo and Rapoport (2008), de Palma and Fosgerau (2013) and others (see, e.g. references within de Palma and Fosgerau, 2011).

The model and experimental design presented in this paper have not been studied 
in this specific form; however, it draws similarities to previous studies. Rapoport et al. (2004) considered a closely related discrete-time model of a queuing system with prespecified opening and closing times, a fixed number of players, fixed service time, service through FIFO, and no early arrivals. The authors assume that when an agent arrives for service he must stay in the queue until either the service is completed or the facility closes, whichever comes first. They define a payoff structure with a common reward for completing the service and a common cost that increases in proportion to the time of waiting. The paper contains numerical mixed strategy solutions for the equilibrium arrival pattern under two selected sets of parameters, which differ in capacity of service for the facility. They report the results of an experimental study with 20 players for a fixed set of parameter values. They find strong support for mixed strategy equilibrium play on the aggregate but not individual level. A companion paper, Seale et al. (2005), studies the same queuing model but allows for early arrivals. The paper computes mixed strategy equilibrium solutions for two selected sets of parameters and verifies them experimentally. The experimental results indicate convergence to equilibrium with experience in playing the game.

This paper differs in a number of important dimensions. First, we explore the welfare implications when employing different queue disciplines within a similar queuing game, while the aforementioned studies establish the strategic behavior and efficiency under FIFO. Second, we consider player utility influenced by two cost factors: impatience for service and queuing time. While the mentioned experimental studies only consider costs of queuing time, other theoretical studies have considered similar cost factors as our paper (e.g. Jain et al., 2011 and Juneja and Shimkin, 2013). Third, we motivate our choice of experimental parameter values based on general predictions of both pure and symmetric mixed strategy equilibrium solutions under all parameter values. Finally, we address fairness perceptions associated with assigned queue disciplines.

\section{The Queuing Game}

The queuing game consists of three players $N=\{A, B, C\}$ who must be served by a single service facility. The facility is open for arrivals within a finite set of periods $T=$ $\{0,1,2, \ldots, k\}$ where 0 denotes the opening period and $k>2$ the latest possible arrival period. The facility is only able to serve one player at each period $t \in T$. Regardless of when the players will arrive, they are admitted to the queue and the facility will complete their service as quickly as possible. Note that the earliest feasible period by which all players can be served is period 2 .

Arrival Strategies. Each player $i \in N$ must simultaneously and independently choose a (mixed) arrival strategy $\alpha_{i} \in \Delta(T)$ where $\Delta(T)$ is the set of probability distributions over the service periods $T$. For clarity, we will refer to $a_{i} \in T$ as a pure strategy arrival of player $i$. Let $\alpha_{i}\left(a_{i}\right)$ denote the probability that arrival strategy $\alpha_{i}$ assigns to $a_{i} \in T$ and 
define the support of $\alpha_{i}$ to be the set of pure strategy arrivals for which $\alpha_{i}\left(a_{i}\right)>0$. We refer to $\alpha=\left(\alpha_{j}\right)_{j \in N}$ as a mixed strategy arrival profile where $\alpha \in \times_{j \in N} \Delta(T)$. Moreover, let $\alpha_{-i}$ denote the list $\left(\alpha_{j}\right)_{j \in N \backslash\{i\}}$ of arrivals in profile $\alpha$ for all players except $i$. Of course, any pure strategy arrival $a_{i}$ can be considered as a mixed strategy that assigns probability 1 to $a_{i}$ and probability 0 to all other strategies. Let $e\left(a_{i}\right)$ denote such a degenerate mixed strategy. We refer to $a=\left(a_{j}\right)_{j \in N} \in \times_{j \in N} T$ as a pure strategy profile.

For analytical convenience, we apply additional notation to describe the arrival characteristics within a strategy profile. First, let an arrival distribution describe a realized arrival periods of a strategy profile $\alpha$. We define the arrival distribution as the number of arriving players at each period from the opening at period 0 until the last player has arrived. For example, $[2,1]$ denotes an arrival distribution with two realized arrivals at period 0 while one at period 1 , and similarly, $[0,1,0,2]$ denotes an arrival distribution with one realized arrival at period 1 while two at period 3 . Note that one arrival distribution can be induced by multiple strategy profiles. Second, for any (symmetric) mixed strategy profile, we sometimes explicitly indicate every pure strategy in support of the profile. For example, if $\alpha^{\prime}$ assigns positive probabilities to period 0 and 2, we denote the profile by $\alpha^{\{0,2\}}$, whereas if $\alpha^{\prime \prime}$ assigns positive probabilities to period 0,1 , and 2 , we denote the profile by $\alpha^{\{0,1,2\}}$.

Queue Discipline. Suppose for some period $t$ that more players arrive at the facility than can be handled. In this case, congestion arises and some players will have to wait until service capacity becomes available.

A queue discipline governs the service priority of the players within the queue. In this paper we consider three disciplines: FIFO, LIFO, and SIRO. We refer to these as the classical queue disciplines. The disciplines are characterized by assigning different priorities to service order: FIFO prioritizes service to players in order of first arrivals, LIFO prioritizes players in order of latest arrivals, while SIRO services players randomly with no regard to order of arrivals. As players choose arrivals in discrete time, the queuing game allows for simultaneous arrivals of players (also referred to as ties). In this case, the players are served with equal probability in accordance to the priority of the queue discipline. For example under FIFO, those who arrive simultaneously are served uniformly over a number of periods until all are served. Under LIFO, however, the players are served uniformly as long as there are no later arrivals. If later arrivals do occur, these arrivals are prioritized for service and the earlier arriving players will not be served before capacity becomes available. Lastly, under SIRO, players are served randomly independent of arrival. The classical queue disciplines are all work-conserving. ${ }^{1}$

Each queue discipline induces a probability mass function, $S_{i t}$, which measures the probability that player $i$ is served at period $t$ given an arrival distribution.

\footnotetext{
${ }^{1} \mathrm{~A}$ queue discipline is work-conserving if the server is never idle when the queue is not empty (see Hassin and Haviv, 2003).
} 
Player Preferences We assume that the players have identical preferences. Each player wishes to be served as early as possible, while minimizing the time spent waiting in the queue. Specifically, we let each player have an initial utility endowment $\gamma$, an impatience cost $\omega t$ for being served at period $t$, and a queuing cost $\delta\left(t-a_{i}\right)$ for waiting in the queue since arrival $a_{i}$. To capture such preferences, we define a player's utility $u_{i}$ as:

$$
u_{i}=\gamma-\omega t-\delta\left(t-a_{i}\right)
$$

where $\gamma, \omega, \delta>0$ are positive constants. Let $\theta=\delta / \omega$ denote the relative queuing cost.

As the service period is (generally) stochastic, we assume that each player aims to maximize her expected utility of (1) with respect to the arrival strategy $\alpha_{i}$. Let the expected utility of player $i$ under profile $\alpha$ be denoted $E U_{i}\left[S_{i t}(\alpha)\right]$. Given the players' preferences specified in (1), the expected utility can be characterized as $E U_{i}\left[S_{i t}(\alpha)\right]=$ $\sum_{a_{i} \in T} \alpha_{i}\left(a_{i}\right) E U\left[S_{i t}\left(e\left(a_{i}\right), \alpha_{-i}\right)\right]=\sum_{a_{i} \in T} \alpha_{i}\left(a_{i}\right) E\left[\gamma-\omega t-\delta\left(t-a_{i}\right)\right]$ which can be rewritten as

$$
E U_{i}\left[S_{i t}(\alpha)\right]=\sum_{a_{i} \in T} \alpha_{i}\left(a_{i}\right)\left(\gamma-\omega E[t]-\delta E\left[t-a_{i}\right]\right)
$$

where $E[t]$ denotes the expected service time and $E\left[t-a_{i}\right]$ the expected queuing time for player $i$ which are induced by the probability mass function $S_{i t}(\alpha)$.

Equilibrium Arrivals. We analyze how specific queue disciplines provide different arrival incentives by comparing their corresponding Nash equilibrium solutions. For any queue discipline, let a Nash equilibrium profile be an arrival profile $\alpha^{*}=\left(\alpha_{i}^{*}, \alpha_{-i}^{*}\right)$ with the property that for every $i \in N$ we have

$$
E U_{i}\left[S_{i t}\left(\alpha_{i}^{*}, \alpha_{-i}^{*}\right)\right] \geq E U_{i}\left[S_{i t}\left(\alpha_{i}, \alpha_{-i}^{*}\right)\right] \text { for all } \alpha_{i} \in \Delta(T)
$$

Thus, no player can (strictly) improve his expected utility by a unilateral arrival deviation. Let $\Phi_{\alpha^{*}}=\sum_{i} E U_{i}$ denote the expected equilibrium welfare of any Nash equilibrium profile. Moreover, we refer to an arrival distribution induced by a pure strategy Nash equilibrium profile as an equilibrium arrival distribution.

Lastly, let player $i$ 's best response function $B_{i}\left(\alpha_{-i}\right)$ be the set of best arrivals given $\alpha_{-i}$, thus $B_{i}\left(\alpha_{-i}\right)=\left\{\alpha_{i} \in \Delta(T): E U_{i}\left[S_{i t}\left(\alpha_{i}, \alpha_{-i}\right)\right] \geq E U_{i}\left[S_{i t}\left(\alpha_{i}^{\prime}, \alpha_{-i}\right)\right]\right.$ for all $\left.\alpha_{i}^{\prime} \in \Delta(T)\right\}$. A Nash equilibrium arrival is an arrival profile $\alpha^{*}$ for which $\alpha_{i}^{*} \in B_{i}\left(\alpha_{-i}^{*}\right)$ for all $i \in N$.

The maximum welfare is the arrival profile that provides no queuing time for any player, i.e. the profiles providing the arrival distribution $[1,1,1]$. However, as players are interested in maximizing their own utility, the equilibrium profile might provide longer queuing times than the optimal as shown in the next section. Let $C=\sum_{i} E_{i}\left[t-a_{i}\right]$ denote the total queuing time which measures the sum of expected queuing times for all players under any pure strategy profile. Intuitively, $C$ measures how the efficiency of a system degrades due to the selfish behavior of its players and coordination issues. 


\section{Equilibrium Analysis}

This section presents the pure and mixed strategy Nash equilibrium arrivals under each of the classical queue disciplines and compares their corresponding equilibrium welfare properties. Section 4.1 describes the analytical method for finding the Nash equilibrium arrivals. Section 4.2 presents the existing equilibrium arrival profiles, their corresponding equilibrium welfare, and the conditions under which they are unique.

\subsection{Method}

We determine the equilibrium arrivals by exploring the players' best responses and establish properties that any equilibrium profile must satisfy (both generally and specific to the queue discipline). Subsequently, we find arrival profiles that satisfy these properties and prove for which relative queuing costs, $\theta$, every such profile is a Nash equilibrium. In the pure strategy analysis, we establish equilibrium arrival distributions rather than individual equilibrium profiles. This is done to conjoin similar welfare outcomes since an arrival distribution provides the same equilibrium welfare as each of its underlying arrival profile permutations. For the mixed strategy analysis, we consider symmetric equilibrium profiles and restrict the analysis only to consider profiles where each player at most randomizes over period 0, 1, and 2. Appendices A.1 and A.2 contain the detailed analysis and proofs used to determine the pure and mixed strategy equilibrium profiles, respectively.

\subsection{Results}

The queuing game provides various equilibrium profiles depending on $\theta$. We outline the existence and uniqueness of pure and symmetric mixed strategy equilibrium profiles and establish the welfare properties across the queue disciplines.

Existence: Table 1 reports the Nash equilibrium profiles under each queue discipline given the relative queuing cost. The equilibrium profiles are illustrated by rows and intervals of $\theta$ by columns. A queue discipline is stated at the intersection in which the equilibrium profile exists. All pure strategy equilibrium profiles are represented by arrival distributions (rows 1-5) while the symmetric mixed strategy profiles by their supported periods (rows 6-8). Table 1 shows that all queue disciplines provide at least one pure or symmetric mixed equilibrium profile for any $\theta$. LIFO is the only discipline that provides both a pure and a mixed equilibrium profile for all $\theta$. There exist no non-degenerated symmetric mixed equilibrium under FIFO and SIRO for $\theta<1$ due to strategic dominance of the pure strategy equilibrium profile [3]. Likewise, FIFO and SIRO provide no pure strategy equilibrium for $1<\theta<3$ and $1<\theta<\frac{5}{3}$, respectively.

Uniqueness: Comparing the coexistence of pure and symmetric strategy equilibrium profiles, Table 1 shows that LIFO do not provide a unique equilibrium profile since there, at least, exists both a pure and symmetric mixed equilibrium for any $\theta>0$. Conversely, FIFO and SIRO provides the unique pure strategy equilibrium profile [3] for any $\theta \in[0,1)$ 
Relative queuing cost $\theta$

\begin{tabular}{|c|c|c|c|c|c|c|c|}
\hline & {$[0,1)$} & 1 & $(1, \sqrt{2}]$ & $\left(\sqrt{2}, \frac{5}{3}\right)$ & {$\left[\frac{5}{3}, 3\right)$} & {$[3,1+2 \sqrt{2}]$} & $(1+2 \sqrt{2}, \infty)$ \\
\hline$[1,1,1]$ & & LIFO & LIFO & LIFO & $\begin{array}{l}\text { LIFO } \\
\text { SIRO }\end{array}$ & $\begin{array}{l}\text { LIFO } \\
\text { SIRO } \\
\text { FIFO } \\
\end{array}$ & $\begin{array}{l}\text { LIFO } \\
\text { SIRO } \\
\text { FIFO } \\
\end{array}$ \\
\hline$[1,2]$ & & LIFO & & & & & \\
\hline$[2,0,1]$ & & $\begin{array}{l}\text { FIFO } \\
\text { SIRO }\end{array}$ & & & & & \\
\hline$[2,1]$ & LIFO & $\begin{array}{l}\text { LIFO } \\
\text { SIRO } \\
\end{array}$ & & & & & \\
\hline [3] & $\begin{array}{l}\text { FIFO } \\
\text { SIRO }\end{array}$ & $\begin{array}{l}\text { FIFO } \\
\text { SIRO } \\
\end{array}$ & & & & & \\
\hline$\alpha\{0,1\}$ & LIFO & LIFO & LIFO & & & & \\
\hline$\alpha^{\{0,2\}}$ & & FIFO & FIFO & FIFO & FIFO & FIFO & \\
\hline$\alpha^{\{0,1,2\}}$ & & & SIRO & $\begin{array}{l}\text { SIRO } \\
\text { LIFO }\end{array}$ & $\begin{array}{l}\text { SIRO } \\
\text { LIFO }\end{array}$ & $\begin{array}{l}\text { SIRO } \\
\text { LIFO }\end{array}$ & $\begin{array}{l}\text { SIRO } \\
\text { LIFO } \\
\text { FIFO } \\
\end{array}$ \\
\hline
\end{tabular}

Table 1: Equilibrium Profiles under FIFO, LiFO, and SIRO

while it provides multiple equilibria for $\theta \geq 1$.

Welfare properties: With the existence of multiple equilibrium profiles, we cannot establish a coherent welfare ordering of the queue disciplines for all values of $\theta$. Nevertheless, we obtain insights into the welfare ordering by analyzing the best and worst case equilibrium welfares of the existing equilibrium profiles. That is, comparing the equilibrium profiles under each discipline that provide the highest and the lowest expected welfare, respectively. Figure 1 depicts the best and worst case equilibrium welfare under each queue discipline given the relative queuing cost $\theta$. The equilibrium welfare $\Phi_{a^{*}}$ is indexed and thus illustrates the rank-orders of the queue disciplines for any set of parameter values.

The best case equilibrium welfare under LIFO is induced by [2,1] for $\theta \in[0,1)$ and $[1,1,1]$ for $\theta \geq 1$, whereas the worst case is induced by $\alpha^{\{0,1\}}$ for $\theta \in[0, \sqrt{2}]$ and $\alpha^{\{0,1,2\}}$ for $\theta>\sqrt{2}$. Under FIFO, the best case is induced by [3] for $\theta \in[0,1),[2,0,1]$ for $\theta=1$, $\alpha^{\{0,2\}}$ for $\theta \in(1,3)$, and $[1,1,1]$ for $\theta \geq 3$, whereas the worst case is induced by [3] for $\theta \in[0,1), \alpha^{\{0,2\}}$ for $\theta \in[1,1+2 \sqrt{2}]$, and $\alpha^{\{0,1,2\}}$ for $\theta>1+2 \sqrt{2}$. Lastly under SIRO,

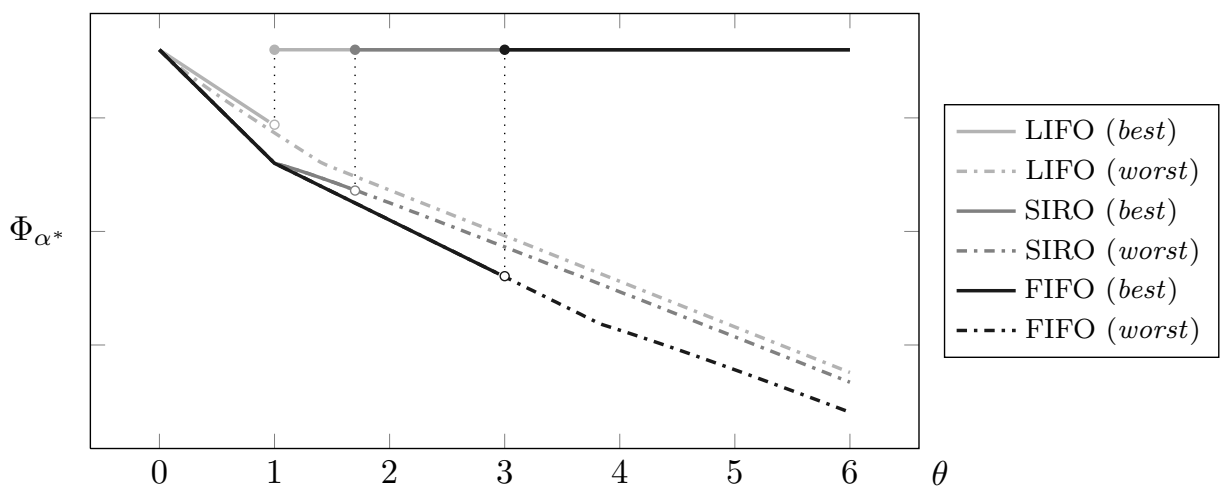

Figure 1: Best and Worst Case Equilibrium Welfare across queue disciplines 
the best case is induced by [3] for $\theta \in[0,1),[2,0,1]$ for $\theta=1, \alpha^{\{0,1,2\}}$ for $\theta \in\left(1, \frac{5}{3}\right)$, and $[1,1,1]$ for $\theta \geq \frac{5}{3}$, whereas the worst case is induced by [3] for $\theta \in[0,1)$ and $\alpha^{\{0,1,2\}}$ for $\theta \geq 1$.

Figure 1 shows that both LIFO's best and worst case equilibrium welfare are strictly higher than FIFO and SIRO's best case equilibrium welfare for $\theta \in[0,1) \cup\left(1, \frac{5}{3}\right)$, whereas they are strictly higher than FIFO's for $\theta \in[0,1) \cup(1,3)$. Moreover, SIRO's best and worst case equilibrium welfare is strictly higher than FIFO's for $\theta \in(1,3)$. Lastly, for all $\theta$, LIFO's worst case equilibrium is strictly higher than FIFO and SIRO's worst case equilibrium, whereas LIFO's best case equilibrium is at least as high as FIFO and SIRO's best case equilibrium.

To summarize, the queue discipline affects the incentives of arrival and thus the corresponding equilibrium welfare. For lower relative queuing costs $\left(\theta \in[0,1) \cup\left(1, \frac{5}{3}\right)\right)$, LIFO generally provides the strictly highest equilibrium welfare among the classical disciplines. Intuitively, LIFO gives players an incentive to smoothen arrivals since the later arrival is prioritized. Conversely, FIFO provides players the incentive to arrive immediately and therefore causes higher congestion, hence lower welfare. For some relative queuing costs $(\theta \in(1,3))$, SIRO provides strictly higher welfare than FIFO and thus provides more smoothing incentives than FIFO (however, still less so than LIFO). To some extent, these welfare properties draw similarities to those of Platz and Østerdal (2012) for a continuum of players.

\section{Experimental Design}

We construct an experimental environment mirroring the theoretical queuing game presented above to evaluate whether the behavior of subjects in our experiment adheres to the theoretical predictions. Specifically, we concentrate on whether people's behavior corresponds to our equilibrium predictions and analyze the causes of observed deviations.

\subsection{Experimental Procedures}

Our experimental setup consists of groups of three subjects that need to be served through a single service facility. The facility becomes active for service at period 0 and can only serve one subject per period. In the experiment, each subject must simultaneously and independently choose an arrival period $a_{i} \in\{0,1,2, \ldots, k\}$ given a preannounced use of queue discipline being FIFO, LIFO or SIRO. When the arrival $a_{i}$ is chosen, each subject is unable to perform any other actions until they receive service at period $t$.

The subjects have the same payoff function represented by the utility function (1) and we let the model parameters be given by $\gamma=480, \omega=50$, and $\delta=40$. The payoff function $\pi_{i}$ for each subject $i$ is thus $\pi_{i}=480-50 t-40\left(t-a_{i}\right)$ for arrival $a_{i}$ and service period $t$. Given that $\theta=\delta / \omega=4 / 5$, the experimental environment is characterized by subjects having slightly higher marginal cost of impatience than queuing time. To avoid 
the possibility of negative payoffs, we restrict the choice of arrivals such that $k=6$. Given the stated parameter values, this restriction does not affect the strategic behavior of subjects since any arrival later than period 6 is strictly dominated by arrivals within period 0 through 3 . Table 2 reports the corresponding predictions prescribed by the pure and symmetric mixed strategy equilibrium arrivals under the classical queue disciplines when $\theta=4 / 5$. These are represented by FIFO*, LIFO* and SIRO*.

The arrival distribution [3] is a unique equilibrium prediction under FIFO and SIRO, while the asymmetric arrival distribution $[2,1]$ and the symmetric mixed strategy profile $\alpha^{\{0,1\}}$ with $\alpha_{i}(0)=2 / 3$ and $\alpha_{i}(1)=1 / 3$ are equilibrium predictions under LIFO. ${ }^{2}$ The symmetry of LIFO's equilibrium predictions thus depends on whether the players' strategy for arrival is a supported mixed or degenerate (pure) strategy. Since the subjects choose their arrivals simultaneously and independently, there are coordination issues associated with obtaining the asymmetric equilibrium profile $[2,1]$. However, by choosing $\theta=4 / 5$, the symmetric mixed strategy equilibrium $\alpha^{\{0,1\}}$ 's probabilities of period 0 and 1 arrivals correspond to an individual randomization of the three pure strategy equilibrium profiles that induce arrival distribution $[2,1]$. To some extent, the two equilibrium predictions are thus compatible when subjects cannot coordinate their arrivals. For the symmetric equilibrium predictions under each queue discipline, the expected queuing time for each subject is 1 under FIFO and SIRO, hence an expected payoff of 390 , while $\frac{19}{27} \approx 0.70$ under LIFO, hence an expected payoff on 400.

Besides aggregate predictions, we also obtain predictions on individual equilibrium behavior through beliefs and their corresponding best responses. We say the subjects hold equilibrium beliefs if they expect the others to arrive in accordance to the equilibrium profiles. Under FIFO and SIRO, the equilibrium beliefs are given by the others both arriving at period 0 and thus are unique and deterministic. Under LIFO, however, the equilibrium beliefs are not unique and may not be deterministic. The theoretical equilibrium beliefs under the symmetric mixed strategy arrival predictions are probabilistic and given by $\alpha_{-i}(0)=2 / 3$ and $\alpha_{-i}(1)=1 / 3$. However, the assumption that subjects are able to formulate well-defined subjective probabilistic beliefs is questionable empirically as well

Table 2: Equilibrium Predictions for $\gamma=480, \omega=50$, AND $\delta=40$

\begin{tabular}{|c|c|c|c|c|c|}
\hline & $\begin{array}{l}\text { Equilibrium } \\
\qquad \alpha^{*}\end{array}$ & $\begin{array}{l}\text { Best Response } \\
B_{i}\left(a_{-i}\right)\end{array}$ & $\begin{array}{l}\text { Equilibrium Beliefs } \\
\qquad a_{-i}^{*}\end{array}$ & $\begin{array}{l}\text { Queuing Time } \\
\qquad E\left[t-a_{i}^{*}\right]\end{array}$ & $\begin{array}{c}\text { Payoff } \\
E\left[\pi_{i}\right]\end{array}$ \\
\hline FIFO* & {$[3]$} & $0, \forall a_{-i}$ & $\{0,0\}$ & 1 & 390 \\
\hline LIFO* $^{*}$ & $\begin{array}{l}\alpha_{i}(0)=2 / 3 \\
\alpha_{i}(1)=1 / 3\end{array}$ & $\begin{array}{l}0, \text { for } a_{-i} \neq\{0,0\} \\
1, \text { for } a_{-i}=\{0,0\}\end{array}$ & $\{0,0\},\{0,1\},\{1,1\}$ & $\frac{19}{27} \approx 0.70$ & 400 \\
\hline SIRO* & {$[3]$} & $0, \forall a_{-i}$ & $\{0,0\}$ & 1 & 390 \\
\hline
\end{tabular}

\footnotetext{
${ }^{2}$ For the choice of parameter values, there exists no other symmetric mixed strategy equilibrium profile than $\alpha^{\{0,1\}}$ even if profiles where players randomize over more periods than period 0,1 , and 2 are considered.
} 
as normatively (Ellsberg, 1961). This gives compelling reason to believe that behavior may not be explained by precise probabilistic beliefs, and it may very well make sense to instead ascribe to players having precise deterministic beliefs about the others. ${ }^{3}$ We say that the deterministic equilibrium beliefs under LIFO are given by $\{0,0\},\{0,1\}$, or $\{1,1\}$. Given the beliefs of the others, each subject has a unique best response arrival. The best response $B_{i}\left(a_{-i}\right)$ is a singleton under FIFO and SIRO given by period 0 (independent of the beliefs concerning $a_{-i}$ ), while it is either 1 (if a subject believes the others arrive at period 0 ) or 0 (for all other beliefs) under LIFO.

The specific parameter values are chosen for several reasons. First, in choosing $\theta=4 / 5$, the experimental environment has a unique equilibrium profile for FIFO and SIRO and a symmetric mixed strategy equilibrium profile under LIFO. Moreover, the environment provides a distinct theoretical difference in the queue disciplines' incentives to arrivals since the expected payoff is higher under LIFO than under FIFO and SIRO. Lastly, $\theta=4 / 5$ ensures compatibility between the pure and symmetric mixed strategy predictions under LIFO as described above.

The experiment involved 6 session with 24 subjects per session. At the beginning of each session, subjects randomly drew a PC terminal number. Each subject then sat down in front of the corresponding terminal and was given printed instructions. After the instructions were read individually, subjects were encouraged to ask questions. To ensure full understanding of the experiment, the subjects had to answer five control questions before the beginning of the actual experiment.

Each session consisted of 3 rounds - one for each of the classical queue disciplines. For each round, we randomly match the subjects into groups of three and announced which queue discipline was assigned for the round. In the subsequent rounds, subjects were randomly rematched into new groups and a new queue discipline was assigned. The random rematching protocol is chosen to minimize spillover effects between rounds.

We choose to let the same subjects participate under each of the three queue disciplines and hereby implement a within-subject experimental design. This design benefits from not requiring a large pool of subjects and helps reduce errors associated with extraneous differences in individual characteristics, as each subject serves as his own baseline. Consequently, it allows us to compare the strategic behavior for each subject when exposed to different queue disciplines. However, drawbacks of the within-subject design are its susceptibility to ordering effects. We control for ordering effects by changing the order in which the different queue disciplines are assigned such that no session has the same order. We have performed a test for potential ordering effects and find no evidence for the existence of such. In addition we also test whether the arrivals under each queue discipline differ significantly across the six session. For each queue discipline, we find no significant arrival differences across the sessions $(p>0.05)$. As a final remark, the within-subject

\footnotetext{
${ }^{3} \mathrm{~A}$ pragmatic argument for subjects having precise deterministic beliefs (rather than probabilistic) is that several experiments finds prevalence of extreme probabilistic beliefs, i.e. degenerate or nearly degenerate beliefs (see Palfrey and Wang, 2009 and references therein).
} 
design causes variable dependency across the rounds since the same subjects serve in more than one. We will take this dependency into account when statistically comparing the queue disciplines.

Each round consists of six stages:

(i) Announcement Stage: The assigned queue discipline is announced.

(ii) Ex-ante Fairness Perception: Half of the subjects state their perceived fairness of the assigned queue discipline by a self-rating scale $f_{\text {ante }}=\{$ strongly fair, fair, neutral, unfair, strongly unfair $\}$.

(iii) Arrival Stage: Subjects are randomly and anonymously matched into groups of three. Subjects announce their individual period of arrival $a_{i}$.

(iv) Beliefs Stage: Subjects announce their deterministic belief regarding the arrival period of the others $a_{-i}$.

(v) Payoff Stage: The server computes individual service times and announces the payoff $\pi_{i}$ for each subject without information about the payoffs of others.

(vi) Ex-post Fairness Perception: Subjects that had not participated in Stage (ii) state their perceived fairness of the queue discipline by $f_{\text {post }}=\{$ strongly fair, fair, neutral, unfair, strongly unfair $\}$.

Stage $(i),(i i i)$, and $(v)$ are the main stages of each round in which subjects receive complete information about the queue system [Stage $(i)]$, process the information to announce their period of arrival [Stage $(i i i)$ ], and receive the payoff [Stage $(v)]$. To limit possible spillover effects to strategic arrivals in subsequent rounds and fairness perception, we exclude any information about the payoffs of the matched others in Stage $(v)$, and moreover, inform subjects that monetary payoffs are randomly based on the performance in one of the three rounds.

In State $(i v)$, subjects state their deterministic beliefs regarding the arrival period of the others. In order to avoid potential hedging effects we do not incentivize subjects belief statements (see e.g. Armantier and Treich (2012) for evidence of potential problems associated with incentivized belief elicitations).

Stage (ii) and (vi) retrieve two separate measures: The perceived fairness ex-ante $f_{\text {ante }}$ [Stage $(i i)]$ and ex-post $f_{\text {post }}$ [Stage $\left.(v i)\right]$ to service. The division of subjects either stating ex-ante or ex-post perception is randomly chosen before the beginning of the three rounds such that no subject participate in both. This is done to mitigate possible consistency effects. Subjects indicate their fairness perception by one of the following statements: strongly fair, fair, neutral, unfair, or strongly unfair. Each statement corresponds to the values $+2,+1,0,-1,-2$ which are treated on ordinal scale of measurement. The scale offers the advantage of retrieving quantitative data which is easy to analyze statistically, however, suffers from possible distortion of issues such as central tendency bias (subjects avoid using extreme response categories), acquiescence bias (compromise to agree with statements as presented) or social desirability bias (agree with statements which portray 
themselves in a more favorable light). See e.g. Norman (2010) for a survey on self-rated scales.

Overall, six independent computerized sessions were conducted in the Laboratory of the Center for Experimental Economics (CEE) at University of Copenhagen on February 13th and October 9th, 2013. We programmed and conducted the experiment with zTree (Fischbacher, 2007). All subjects were students from the University of Copenhagen. No subject was used in more than one session, yielding 144 subjects. Each session lasted approximately 1.5 hours. Instructions, data and zTree program are available upon request.

\subsection{Hypotheses}

Given the experimental design above, we analyze to what extent the equilibrium predictions in Table 2 correspond to the behavior observed in the experiment. To do so, we propose five hypotheses that collectively test whether subjects adhere to aggregate and individual equilibrium behavior.

Aggregate behavior is examined by comparing the arrival distribution observed in the experiment with the distribution prescribed by the equilibrium profiles. We form our hypothesis of aggregate behavior based on the predictions in Table 2.

Hypothesis 1. Subjects arrive in accordance to the symmetric equilibrium profiles under FIFO, SIRO, and LIFO.

Symmetric arrival strategies eliminate coordination issues associated with obtaining the equilibrium profiles. Following Hypothesis 1, each subject will in equilibrium arrive at period 0 under FIFO and SIRO, while randomize over period 0 with probability $2 / 3$ and over period 1 with probability $1 / 3$.

Individual equilibrium behavior requires that the subjects arrive in accordance to their best response arrivals. We examine this by comparing each subject's arrival to their corresponding best response arrival $B_{i}\left(a_{-i}\right)$ given her deterministic beliefs $a_{-i}$.

Hypothesis 2. Subjects arrive in accordance to their best response arrivals $B_{i}\left(a_{-i}\right)$.

Hypothesis 2 is based on the assumption that each subject is payoff-maximizing. ${ }^{4}$ If evidence favors Hypothesis 2 under FIFO and SIRO, Hypothesis 1 follows immediately due to the unique and symmetric individual equilibrium behavior. However, this it not necessarily the case under LIFO since multiple equilibrium beliefs exist. If evidence favors both Hypothesis 1 and 2 under LIFO, we conclude that subjects are payoff-maximizing and hold correct beliefs. Conversely, if evidence favors Hypothesis 2 while not Hypothesis 1 , we conclude that subjects are payoff-maximizing, however do not have correct beliefs.

In the case that subjects do not adhere to equilibrium behavior under any of the queue disciplines, we are interested in examining whether the subjects still obtain the shortest

\footnotetext{
${ }^{4}$ Indisputably, this assumption has been proven inaccurate in many games (Tversky and Kahnemann, 1974, and experiments Grether and Plott, 1979), however, our experiment was designed to reduce computational complexity of subjects' best response arrival; hence, mitigate potential non-maximizing behavior.
} 
expected queuing time and highest expected payoff under LIFO compared to FIFO and SIRO as it is predicted by theory.

Hypothesis 3. Subjects arrive such that the expected queuing time is shorter under LIFO compared to under FIFO and SIRO.

Hypothesis 4. Subjects arrive such that the expected payoff is higher under LIFO compared to under FIFO and SIRO.

Hypotheses 3 and 4 are based on predictions about the strategic behavior under LIFO. Given the priority of latest arrivals, LIFO provides subjects with stronger incentives to smoothen arrivals relatively to FIFO and SIRO. Subjects will cause shorter expected queuing times and thus higher expected payoff if they respond to such strategic incentives.

In addition to testing whether subjects adhere to equilibrium behavior, we examine the perceived fairness of the classical queue disciplines. In our setup, players' payoffs do not include fairness concerns but only consider costs of impatience and queuing time. Consequently, we make an implicit assumption of no fairness differences across the classical queue disciplines or, at least, that fairness concerns do not influence utility. We test this assumption by the following hypothesis.

Hypothesis 5. Subjects perceive the queue disciplines to be equally fair.

The result of Hypothesis 5 is important for several reasons. First, if evidence shows that subjects perceive fairness differently across the queue disciplines, this would call into question whether (and how) these concerns are taken into consideration in the subject's strategic choice of arrival. Second, if fairness perceptions, supposedly, impose (dis)comfort when participating in queues, it questions whether (and how) to account for such when measuring welfare.

\section{Experimental Results}

In this section we test Hypothesis 1 through 5. The statistical analysis follow the convention that a significance level of 5 percent or less is significant. For all results presented in the section below, their test statistics can be seen in Appendix A.3, p. 33, Table 3.

\subsection{Arrival Distribution}

Figure 2 reports the arrival distribution observed in the experiment. The distribution is illustrated as the proportion of arrivals at period 0 throughout 6 under each queue discipline. In order to compare the observed arrival distribution to the predicted arrivals in Table 2, we display the corresponding predicted proportion in equilibrium by the shaded bars and labeled FIFO*, LIFO* and SIRO*.

The arrival distributions in Figure 2 show that subjects arrive differently depending on the queue discipline. There is a high incidence of immediate arrivals at the opening period 


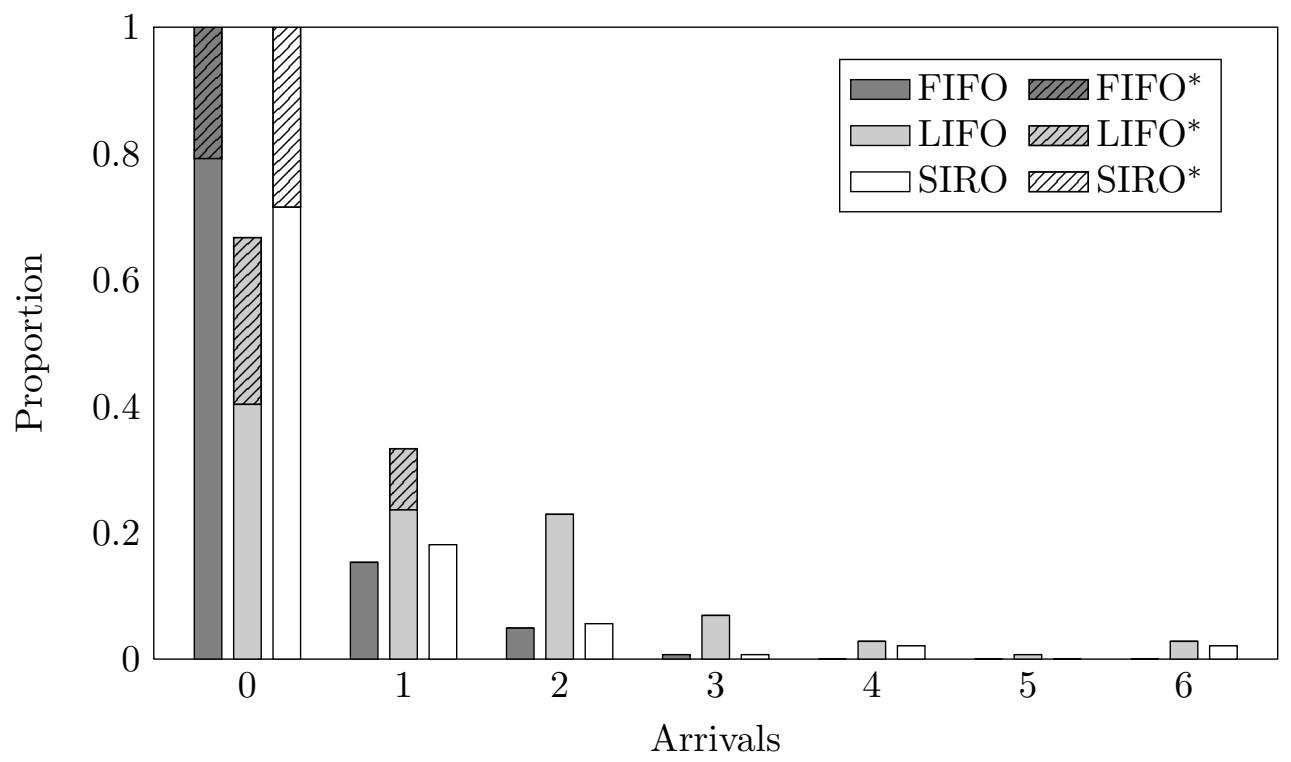

Figure 2: Arrival Distribution Across Queue Disciplines

0 under FIFO and SIRO, while a greater dispersion of arrivals under LIFO. Under FIFO and SIRO, the proportion of immediate arrivals are 0.79 and 0.71 , respectively, and are followed by low proportions of arrivals in the subsequent periods, while 0.40 under LIFO are followed by a nearly identical proportion of arrivals at period 1 and 2 . In regards to arrival variance, higher dispersion is observed under LIFO and SIRO since a proportion of subjects arrive very late. That is, a proportion of 0.06 arrive later than period 3 under LIFO, while 0.05 arrive later than period 3 under SIRO. ${ }^{5}$

We use the one-sided Wilcoxon-Mann-Whitney (WMW) test to compare the distributional differences between the experimental arrivals and the equilibrium predictions where FIFO* $^{*}$ and SIRO* have all subjects arriving immediately at period 0 , while LIFO* has $2 / 3$ of the subjects arrive at period 0 and $1 / 3$ in the subsequent period 1 . Evidence suggests that subject do not adhere to the aggregate arrival predictions $(p<0.01)$ under any of the classical queue disciplines. We therefore reject Hypothesis 1. However, when comparing the queue disciplines' observed arrival distributions to each other, we find that subjects arrive significant later under LIFO compared to FIFO and SIRO $(p<0.01)$, whereas there is no statistical differences in arrivals under FIFO and SIRO $(p>0.05)$. This is supported by the one-sided Wilcoxon signed-rank test. To some extent, this strategic behavior leans towards the theoretical intuition.

\subsection{Best Response Arrivals}

To test Hypothesis 2, we analyze whether subjects arrive in accordance to their best response arrival. Recall from Table 2 that the best response under FIFO and SIRO is

\footnotetext{
${ }^{5}$ In fact, we recorded five attempts to arrive later than the latest accepted period 6 (three under LIFO and two under SIRO). As we do not allow for later arrivals than period 6 in the experiment, a programmed message alert asked the subjects to choose an arrival period earlier than period 6 .
} 
period 0 while 1 or 0 under LIFO. It follows immediately that any arrivals that differ from 0 under FIFO and SIRO, while 0 and 1 under LIFO, cannot be best responses. Figure 3 reports the proportion of subjects that arrive in accordance to the best response under each queue discipline. The highest proportion of best response arrivals are observed under FIFO (0.79) and SIRO (0.72), while distinctly lower under LIFO (0.40). However, as the best response under FIFO and SIRO are independent of beliefs and equal to the opening period, these observed proportions might be exposed to upwards bias as it potentially captures non-intentional best response arrivals.

The Fisher exact test is applied to compare the observed proportion of best response arrivals with the predicted proportion of $100 \%$ best responses. Evidence suggests that a significant proportion of subjects do not adhere to best response arrivals $(p<0.01)$ under any of the classical queue disciplines. We therefore reject Hypothesis 2. We apply the onesided McNemar change test to compare the relative proportions of best response arrivals across the queue disciplines. We find no difference in the proportions under FIFO and SIRO $(p>0.10)$, whereas LIFO provides a significant lower proportion of best response arrivals compared to FIFO and SIRO $(p<0.01)$.

To go one step further, we examine the distance between the observed and corresponding best response arrival. We define the distance by $d=\left|a_{i}-B_{i}\left(a_{-i}\right)\right|$ and compute $d$ across all subjects. Figure 3 reports the distribution of distances under all queue disciplines illustrated by the stacked gray toned bars. The distribution of distances follows the dispersion we observed in Figure 2. Specifically under LIFO and SIRO, the distances span between 1 and 6 periods yielding a standard deviation on 1.4 (LIFO) and 1.1 (SIRO), while the distance primarily spans between 1 and 2 periods under FIFO yielding a lower standard deviation of 0.6. When comparing the average distances across the queue disciplines, we find that subject under LIFO arrive with greater distance to the best response

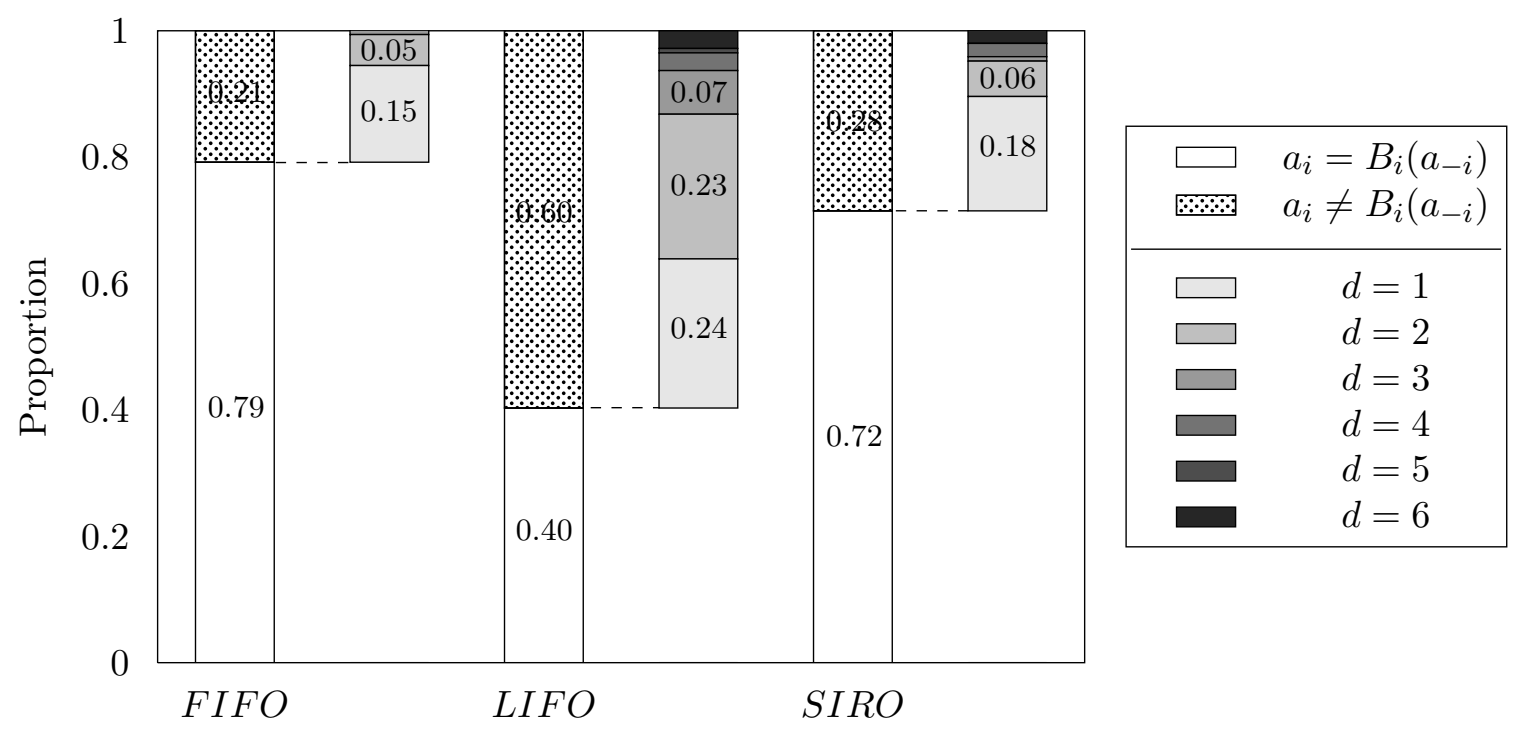

Figure 3: Best Response Arrivals Across Queue Disciplines 
compared to subjects under FIFO and SIRO.

The final point of interest is to examine whether subjects believe others to behave according to payoff-maximization and whether coordination issues cause deviations from aggregate equilibrium behavior. This is done by comparing the subjects' stated beliefs to the equilibrium beliefs stated in Table 2. We find that a high proportion of subjects do not hold equilibrium beliefs: $53 \%$ and $47 \%$ of subjects had equilibrium beliefs $\{0,0\}$ under FIFO and SIRO, respectively, while $46 \%$ under LIFO had equilibrium beliefs $\{0,0\}$, $\{0,1\}$, or $\{1,1\}$. Moreover, $32 \%$ of subjects believe others would arrive later than period 2 under LIFO. If we alternatively look at the beliefs of subjects who arrived according to their best response, the proportions of equilibrium beliefs increase under LIFO (59\%), while barely under FIFO (54\%) and SIRO (53\%).

\subsection{Expected Queuing Times}

Having rejected Hypothesis 1 and 2, we are interested in testing whether LIFO still provides shorter queuing times than FIFO and SIRO. We test Hypothesis 3 by examining the distribution of expected queuing times across the observed arrivals. In the experiment, subjects are randomly matched into groups of three. The distribution of queuing times is determined by the arrival distribution within each group and service priority of the queue discipline. Given that no subject can coordinate nor communicate with their grouped others, potential miscoordination of arrivals might induce longer queuing times than otherwise. We adjust for such issues by computing the expected queuing time under each arrival period. That is, the average queuing time under every possible group match (given the choice of arrival period), weighted by the probability of each group match's occurrence.

Figure 4 reports the distribution of expected queuing times across all queue disciplines along with the corresponding equilibrium predictions. The distributions are illustrated by box and whisker plots. The box represents the ranges of the 25 th and 75 th percentile of expected queuing times, while the whiskers extend to the upper and lower adjacent values (within 1.5 times the interquartile range). The circles represent outside values that fall outside the adjacent values. The bold lines within each box represent the corresponding median of expected queuing times. FIFO*, LIFO* and SIRO* denote the distribution of queuing times in equilibrium.

Figure 4 shows substantial differences in the distribution of expected queuing times between both disciplines and equilibrium predictions. First, comparing across the queue disciplines, the majority of subjects under FIFO obtain longer expected queuing times than the other disciplines, while subjects under LIFO obtain the lowest. In fact, 123 subjects have longer expected queuing times under FIFO than under SIRO, while 137 subjects compared to under LIFO. The median expected queuing time is 0.79 under FIFO, 0.78 under SIRO and 0.29 under FIFO. The one-sided signed-rank test supports the differences as it rejects the hypothesis of no difference between expected queuing times under all 


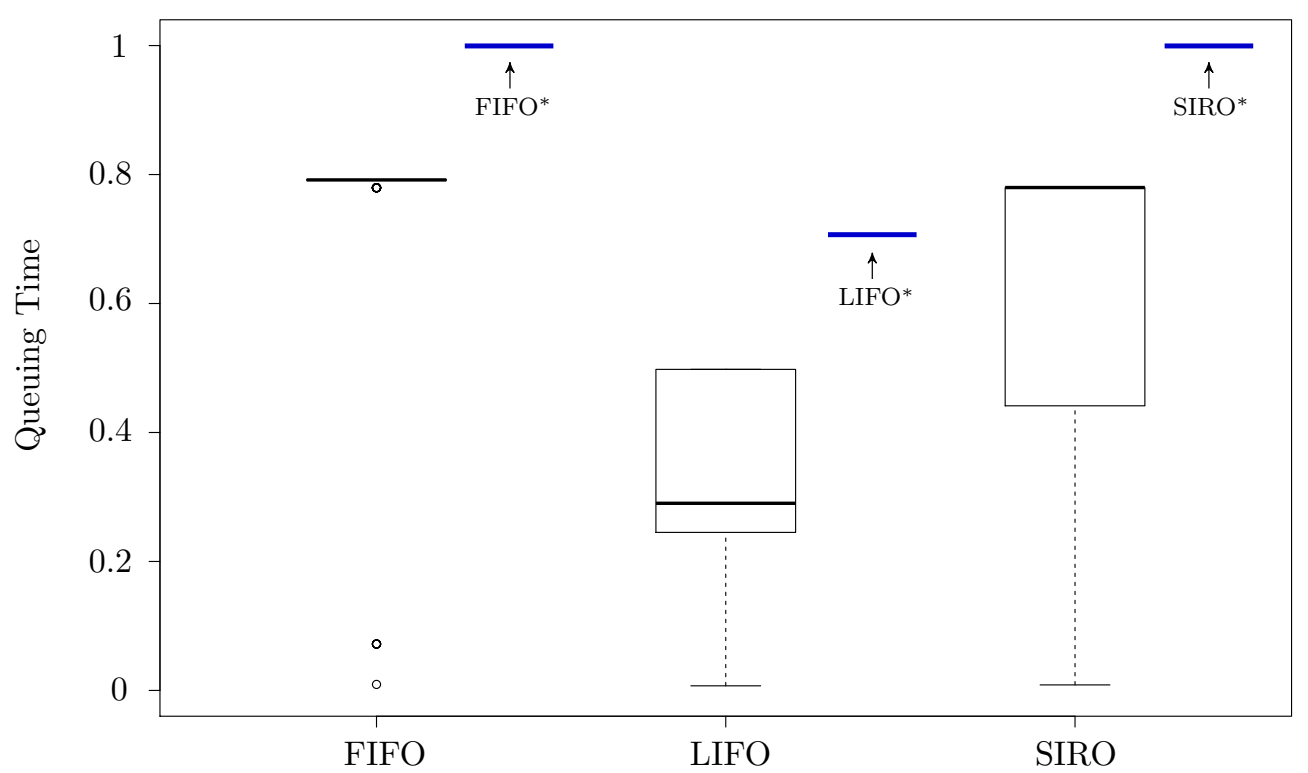

Figure 4: Expected Queuing Time Distribution Across Queue Disciplines

queue disciplines $(p<0.01)$. We therefore accept Hypothesis 3. Second, compared to the equilibrium predictions, subjects obtain shorter expected queuing times under all queue disciplines than prescribed by their corresponding equilibrium predictions. This is supported by the one-sided WMW test.

The observed differences in expected queuing times are unsurprising given the arrival distribution from Figure 2. The high proportion of immediate arrivals under FIFO and SIRO cause high level of congestion and thereby longer expected queuing times. As some subjects arrive later under SIRO compared to FIFO, congestion is reduced within groups containing these subjects and hence causes an overall reduction in expected queuing times. Similarly, the high dispersion of arrivals under LIFO cause low congestion within groups and therefore reduces expected queuing times substantially. Similar arguments explain the differences in equilibrium queuing times.

\subsection{Expected Payoff}

What about the distribution of expected payoffs? Recall subjects are obtaining payoffs depending on the period of service and queuing time. To adjust for potential miscoordination biases, we compute the expected payoffs for all subjects given their arrival period and expected queuing times.

Figure 5 reports the distribution of expected payoffs across queue disciplines along with the corresponding equilibrium predictions. The distributions are illustrated by similar box and whisker plots as for the queuing time distribution. FIFO*, LIFO* and SIRO* denote the expected payoff distribution prescribed by the equilibrium predictions under each discipline.

There is clear indication of differences in the variance of expected payoffs across the 


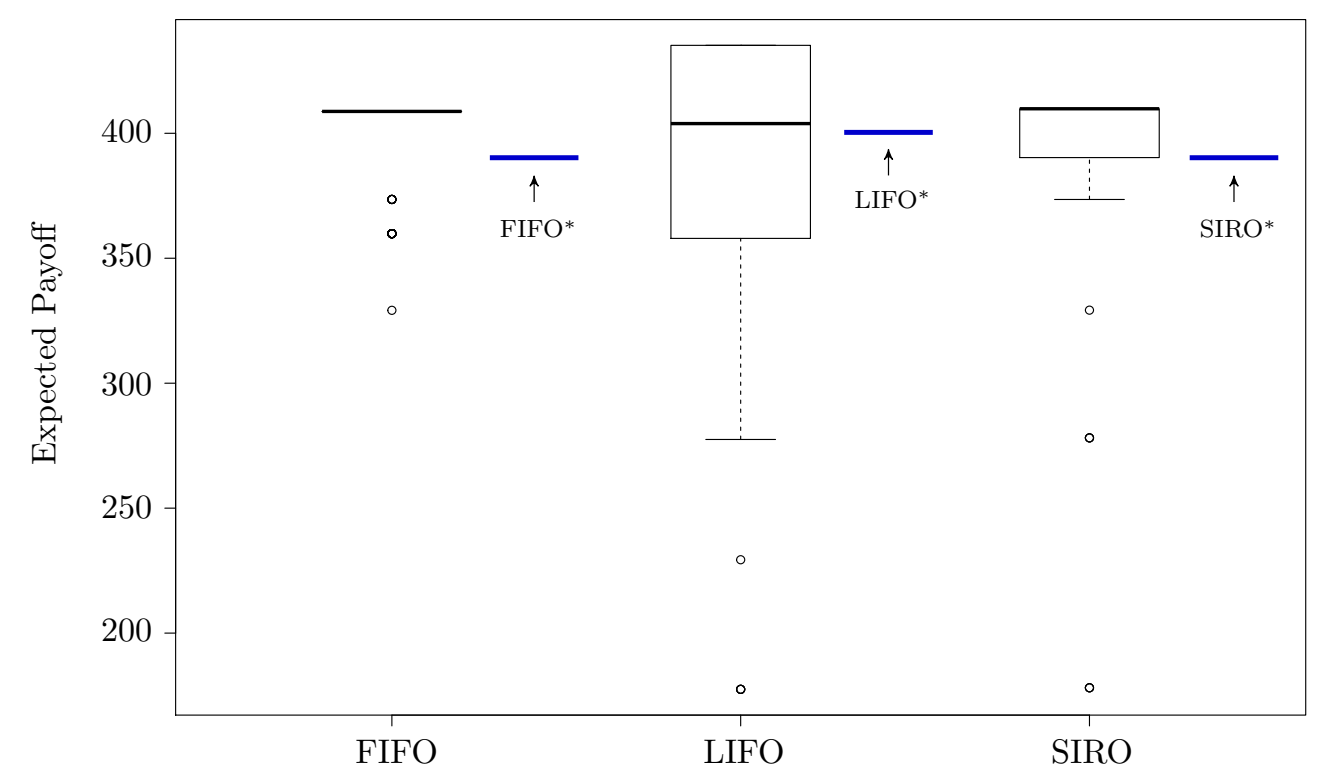

Figure 5: Expected Payoff Distribution Across Queue Disciplines

disciplines. Subjects under LIFO obtain the highest variance, while the lowest under FIFO. The medians of expected payoffs across disciplines are relatively close being 408.8 under FIFO, 403.9 under LIFO and 409.8 under SIRO. The one-sided signed-rank test was used to test the distributional differences in expected payoffs among the queue disciplines. We find that LIFO obtain the same expected payoff as under the other disciplines $(p>0.05)$, whereas subjects obtain significant higher expected payoffs under SIRO compared to under FIFO $(p<0.01)$. This results is explained by a combination of higher expected payoffs for period 0 arrivals under SIRO (409.8) than under FIFO (408.8) and similar high proportions of period 0 arrivals (FIFO: 0.79, SIRO: 0.71). Consequently, 113 subjects under SIRO have higher expected payoffs than under FIFO; hence the statistical significance. Following these findings, we reject Hypothesis 4.

Similarly to the three analyses above, the expected payoff distributions do not correspond to the equilibrium predictions. The WMW test was used to compare the distributional differences between the expected payoffs observed and those in equilibrium. Evidence suggests that subjects obtain significant higher expected payoffs in the experiment compared to those in equilibrium $(p<0.01)$ under all classical queue disciplines. In fact, 114 subjects under FIFO, 92 under LIFO, and 129 under SIRO have higher expected payoffs compared to the equilibrium predictions. The reason why subjects successfully outperform the equilibrium in regards to expected payoff is that they reduced the expected queuing time by leveling out their arrivals. Instead of immediate arrival as prescribed by the equilibrium solution, subjects arrived later and thereby reduced congestion. On the flip side, these late arrivals are also what caused subjects under LIFO not to yield higher expected payoffs than under FIFO and SIRO, as they arrived "too" late such that the impatience cost exceeded the gain in payoff from significantly shorter queuing times. 


\subsection{Fairness Perceptions}

Beyond analyzing adherence to equilibrium behavior, we also examine how subjects perceive the fairness of each queue discipline. As we will show below, the analysis suggests that subjects do indeed perceive the disciplines significantly different in regards to fairness. Specifically, FIFO is perceived to be most fair, while LIFO the least fair.

In the experiment, subjects announce their fairness perception either ex-ante to service, $f_{\text {ante }}$, or ex-post, $f_{\text {post }}$. Figure 6 reports the announced perceptions across the queue disciplines. For ex-ante perceptions [Figure 6 (a)], most subjects perceive FIFO to be within the fair categories, while LIFO and SIRO within the unfair categories. Specifically, the average fairness perception of FIFO is between fair and strongly fair (numerical 1.4), around unfair (-0.7) for SIRO, and lastly between unfair and strongly unfair (-1.4) for LIFO. In addition Figure 6 (a) shows that subjects have higher dispersion in fairness perception under SIRO and LIFO than under FIFO. For ex-post perceptions [Figure 6 (a)], subject reveal similar distribution of fairness perception as ex-post, however, the average fairness perceptions of SIRO and LIFO are relatively more fair, whereas approximately the same for FIFO.

The one-sided signed-rank test was used to test the distributional differences in perceived fairness among the queue disciplines when measured either ex-ante and ex-post,

(a) Ex-ante Perception

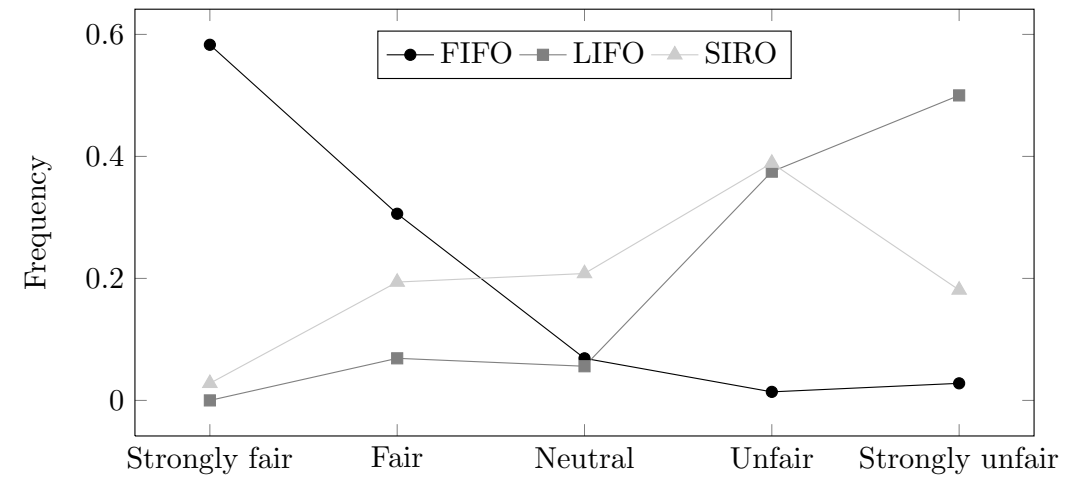

(b) Ex-post Perception

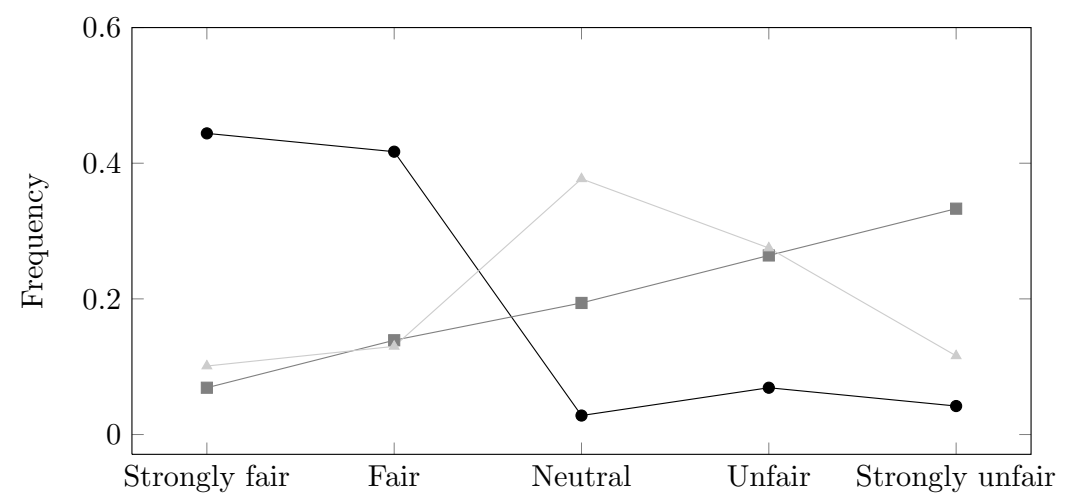

Figure 6: Fairness Perception across Queue Disciplines 
respectively. Evidence suggests that subjects perceive FIFO as the most fair queue discipline, while LIFO the least fair $(p<0.01)$. This is consistent under both ex-ante and ex-post perceptions. We therefore reject Hypothesis 5. In addition we test whether the fairness perceptions of a specific queue discipline differs across ex-ante and ex-post perceptions. Differently from the previous tests, the samples are independent as we asked different subjects to announce either ex-ante or ex-post perception. Given the independence of samples, we apply the WMW test under the null that the distribution of ex-ante and ex-post fairness perceptions are the same under each queue discipline. We find find that under LIFO and SIRO there exists a difference between ex-ante and ex-post perceptions, while we cannot reject that the fairness perceptions under FIFO are the same ex-post and ex-ante. The perceived fairness of LIFO and SIRO is higher when measured ex-post compared to ex-ante.

\section{Interpretation and Discussion}

Queuing theory and mechanism design widely recognizes the economic imperative of designing queuing systems such that their procedural characteristics induce optimal strategic behavior. In this paper, we present experimental evidence suggesting that the use of specific queue disciplines affects the strategic behavior of the players and hereby the level of congestion within a queuing game. We find that subjects arrive for service with greater dispersion when participating under the LIFO discipline, whereas they tend to arrive immediately under FIFO and SIRO. As a consequence, lower levels of congestion are obtained under LIFO as compared to FIFO and SIRO. To some extent, this strategic behavior is consistent with our theoretical intuition. However, the experimental arrivals do not comply with the behavioral predictions prescribed by the Nash equilibrium as a significant proportion of the subjects do not behave according to individual payoff maximization. This non-optimal behavior is observed under all queue disciplines but seems to be particularly strong under LIFO. While the observed behavior under LIFO causes lower congestion, the subjects do not obtain higher expected payoffs because higher costs of impatience from arriving "too" late exceed the gains of shorter expected queuing times. The theoretical prediction of higher welfare under LIFO is thus not recovered experimentally. Interestingly, however, subjects obtain higher median expected payoffs in the experiment than that prescribed by equilibrium. The reason is that the proportion of (later) non-optimal arrivals causes lower levels of congestion; hence higher expected payoffs for subjects.

In addition this paper also studied the perceived fairness of the queue disciplines. We find that subjects perceive the fairness of queue disciplines significantly different, i.e. FIFO is perceived the most fair while LIFO the most unfair. These perceptions are consistent whether stated ex-ante or ex-post to the receipt of payoffs. While LIFO provides shorter queuing times and not significantly different payoffs than FIFO and SIRO, surprisingly perhaps, subjects still perceive LIFO to be less fair. This result could have profound implications for the accuracy of our theoretical predictions as player utilities do not include 
fairness concerns. That is, if fairness concerns are highly important in queues (sometimes more important than the duration of queuing time, cf. Rafaeli et al., 2002, 2005), then the perceived unfairness of the LIFO discipline might affect the individual utilities negatively and thus lower the welfare compared to FIFO and SIRO. A social planner must therefore take fairness concerns into account when choosing the welfare optimal queue discipline.

Existing literature partly supports our finding of discrepancies between theoretical predictions and experimentally observed outcomes. For a wide range of games unspecific to queuing, experimental participants do not consistently behave in accordance to payoffmaximizing behavior as prescribed by the Nash equilibrium (see e.g. Fudenberg and Levine, 1998 for theoretical studies and Camerer (2003) for experimental). Specific to queuing games, however, a closely related paper by Rapoport et al. (2004) finds no support for equilibrium play on the individual level whereas the aggregate behavior corresponds to their mixed strategy equilibrium predictions. While the non-optimal individual behavior corresponds to our results, the accuracy of predicted aggregate behavior differs. This discrepancy is surprising since our theoretical predictions under FIFO involve a symmetric and unique pure strategy Nash equilibrium with strategic dominance as opposed to the relatively more complex mixed strategy equilibrium in Rapoport et al.'s experiment. Indeed, it may be argued that repeating our game would allow for belief learning and thereby have aggregate behavior converge to the equilibrium predictions. While such learning effects would be interesting to explore in future research, we think it is interesting that the observed social outcome is still better than the equilibrium predictions in a one-shot game, despite the fact that many subjects fail to arrive in accordance to best responses (some even quite far from it).

The complexity of modeling cognitive and emotional determinants on agent's strategic decisions leaves economic literature with insufficient evidence for explaining why agents fail to consistently behave according to payoff-maximization. A possible explanation (unspecific to queuing) is suggested by Conlisk (1996), who argues that bounded rationality can cause systematic mistakes by using "heuristic" decision-making, or rule of thumb, which fails to capture the full logic of a decision. In continuation of this idea, Camerer (2003) surveys expected utility models that describe human decision-making better than the standard model. In particular, he claims that two major systematic deviations from expected payoff maximization are consistently observed in people: risk aversion (where players dislike risk and avoid outcomes where risk relative to expected gains are overweighed) and non-linear decision weights (where prospects are not directly weighted by their probability of occurring, but rather by some non-linear decision weight instead). In regards to risk aversion, we examine whether the existence of such would alter the equilibrium predictions for our experiment. To do so, we respecify the players' utility function (1) as a concave function and thereafter recalculate the corresponding equilibrium predictions. We consider the concave utility function $\tilde{u}_{i}=\left(u_{i}\right)^{k}$ where $k \in[0,1]$ denotes the degree of risk aversion, i.e. low values of $k$ indicate high risk aversion and vice versa. Given the chosen experimental parameters $(\gamma=480, \omega=50, \delta=40)$, we find that [3] is a unique 
equilibrium arrival distribution under FIFO and SIRO for any $k \in[0,1]$. Thus, no degree of risk aversion will alter the experimental equilibrium predictions. Similarly, the arrival distribution $[2,1]$ is a unique equilibrium for any $k>0.05$ under LIFO, however, for the special case of excessive risk aversion $(0<k \leq 0.05)$, players will deviate from the $[2,1]$ and no equilibrium exists. Given the restricted interval in which no equilibrium exists, it is reasonable to conclude that risk aversion does not alter the equilibrium predictions within our experiment, and thus cannot account for the observed equilibrium deviations. Future studies could extend the research on strategic queuing games by testing similar systematic behavioral deviations and test their accuracy in predicting strategic arrivals.

We also provide some reflections on the observed fairness results. The analysis identifies two major characteristics: First, the fairness perception differs significantly across queue disciplines, and second, ex-ante and ex-post fairness perception differs significantly within each queue discipline. The two results suggest that perceived fairness depends on determinants which differ in ex-ante and ex-post perception. We believe that ex-ante fairness perceptions are solely based on procedural characteristics of the queue discipline, whereas ex-post perceptions are adjustments to the ex-ante perceptions after individual payoff realizations. In regards to ex-ante perception, many studies have suggested procedural fairness determinants associated with a queue discipline (see Avi-Itzhak et al., 2011 for an excellent survey and references therein). Moreover, empirical evidence on the importance of fairness in queues is provided by Rafaeli et al. $(2002,2005)$ who shows that fairness is highly important for customers waiting under various queuing and scheduling policies. Highly related to the present queuing game, Avi-Itzhak and Levy (2004) propose a fairness measure that ranks the fairness of various queue disciplines when players are homogenous and have identical service times. The paper assumes several elementary axioms on fairness and shows, in steady state, that the waiting time variance under each queue discipline can serve as a surrogate for its unfairness measure. The measure thus implies that the most fair queue discipline is FIFO while the most unfair is LIFO. ${ }^{6}$

The same fairness order is observed in our experimental results. While fairness determinants for ex-ante perception are well-recognized and seem to support our findings, the determinants for ex-post perception have received limited attention. We believe selfcentered inequity aversion (Fehr and Schmidt, 1999), and/or disappointment (Bell, 1985) could be possible behavioral characteristics that explain the ex-post adjustment in fairness perception. The argument is that subjects have psychological reactions to an payoff outcome (or distribution) that does not match up to expectations. This reaction may well influence the ex-post fairness perception of the queue discipline. The validity of such ex-post fairness determinants remains a behavioral issue for future research.

\footnotetext{
${ }^{6}$ The fairness property follows from Kingman (1962) who shows that FIFO minimizes the variance of waiting time among all work-conserving and uninterrupted queue disciplines while LIFO maximizes the variance.
} 


\section{A Appendix}

\section{A.1 Pure strategy equilibrium analysis}

This section presents the pure strategy Nash equilibrium arrival profiles under each of the classical queue disciplines and establish their corresponding equilibrium welfare.

\section{Auxiliary Results}

We first make some observations about a pure strategy equilibrium profile that apply for all queue disciplines:

Lemma 1. Let profile $a^{*}$ be a pure strategy Nash equilibrium under any queue discipline. Then $a^{*}$ has all players served at period 2

Proof. We prove by contradiction. Let $r_{t}$ denote the number of players arriving at period $t$. Suppose that an arrival profile $a^{\prime}$ is characterized by $\sum_{t=0}^{t^{\prime}} r_{t}<t+1$ for some $t^{\prime} \in\{0,1,2\}$, i.e. no player is served at $t^{\prime}$ despite available capacity at the facility. This implies that at least one player $i \in N$ is served at some later period $t^{\prime \prime}>2$. With available service capacity at $t^{\prime}$, the player served at $t^{\prime \prime}$ could instead arrive at $t^{\prime}$ and be served immediately without queuing time. Given strictly increasing impatience costs with $\delta>0$, it follows that the player obtains higher expected utility by arriving at $t^{\prime}$. Conclusively, a Nash equilibrium profile must have $\sum_{t} r_{t} \geq t+1$ for every $t \in T$, such that the last player is served at period 2 .

Lemma 2. Let profile $a^{*}$ be a pure strategy Nash equilibrium characterized by the total queuing time $C^{*}$ and another equilibrium profile $a^{\prime}$ characterized by $C^{\prime}$. It follows that any difference between the equilibrium welfare $\Phi_{a^{*}}$ and $\Phi_{a^{\prime}}$ is caused by differences in $C^{*}$ and $C^{\prime}$. Thus, if $C^{*}=C^{\prime}$ then $\Phi_{a^{*}}=\Phi_{a^{\prime}}$, while if $C^{*}<C^{\prime}$ then $\Phi_{a^{*}}>\Phi_{a^{\prime}}$.

Proof. Let profile $a=\left(a_{i}, a_{-i}\right)$ be a Nash equilibrium. Given identical preferences of players, we may write the expected equilibrium welfare $\Phi_{a}$ as

$$
\begin{aligned}
\Phi_{a^{*}} & =\sum_{i} E U\left[S_{i t}\left(a_{i}, a_{-i}\right)\right] \\
& =\sum_{i}\left(\gamma-\omega E[t]-\delta E\left[t-a_{i}\right]\right) \\
& =\sum_{i} \gamma-\omega \sum_{i} E[t]-\delta \underbrace{\sum_{i} E\left[t-a_{i}\right]}_{=C}
\end{aligned}
$$

where $\gamma, \omega, \delta$ are constants, $\sum_{i} E[t]$ denotes the aggregated expected service time, and $C$ the total queuing time.

We prove that only $C$ can cause differences in expected welfare between two equilibrium profiles since $\sum_{i} E[t]$ is constant under any equilibrium profile. This is proven by the following two observations: First, it follows by Lemma 1 that any equilibrium profile has the last player served at period 2, thus the facility uses its full service capacity within the periods $\{0,1,2\}$. Consequently, in equilibrium, the probability that one player is served 
at each period $t \in\{0,1,2\}$ equals 1 , thus $\sum_{i} S_{i t}=1$. Second, given $E[t]=\sum_{t}\left(t \cdot S_{i t}\right)$, we may write the aggregated expected service time as

$$
\begin{aligned}
\sum_{i} E[t] & =\sum_{i} \sum_{t}\left(t \cdot S_{i t}\right) \\
& =\sum_{t}\left[\sum_{i} S_{i t} \cdot \sum_{i} t\right] \\
& \stackrel{(*)}{=} \sum_{t} t=3
\end{aligned}
$$

where operation $(*)$ applies $\sum_{i} S_{i t}=1$ and that $t$ is independent of each player, i.e. $\sum_{i} t=t$. Conclusively, the aggregated expected service time is constant and therefore independent of any Nash equilibrium profile. That is, only a difference in $C$ can provide welfare differences between two Nash equilibrium profiles.

Let profile $a^{*}$ be a Nash equilibrium characterized by the total queuing time $C^{*}$ and profile $a^{\prime}$ another equilibrium characterized by $C^{\prime}$, it then follows immediately that $\Phi_{a^{*}}=\Phi_{a^{\prime}}$ when $C^{*}=C^{\prime}$ and $\Phi_{a^{*}}>\Phi_{a^{\prime}}$ when $C^{*}<C^{\prime}$.

In the following, we examine the pure strategy arrival profiles for each of the classical queue disciplines.

\section{First-In First-Out (FIFO)}

We make some observations about a pure strategy Nash equilibrium profile under FIFO.

Lemma 3. Let $a^{*}$ be a Nash equilibrium under FIFO. Then it follows for $a^{*}$ that

(i) The number of arriving players never exceeds the service capacity for any period $t>0$.

(ii) If all players do not arrive at period 0, then the last player arrives at period 2 and is served immediately without queuing time.

Proof. Part (i) and (ii) are proven separately.

First for part $(i)$ we prove by contradiction: Suppose an equilibrium profile $a^{\prime}$ has the number of arrivals exceeding the service capacity (i.e. $r_{t^{\prime}}>1$ ) for some $t^{\prime}>0$. Excluding all profiles that do not satisfy Lemma 1 , only one profile has $r_{t^{\prime}}>1$ for some $t^{\prime}>0$; namely $[1,2]$. With strictly increasing impatience cost over $t$, one of the players arriving at period 1 could increase her expected utility by arriving at period 0 instead, since she would obtain an earlier expected service time for the same expected queuing time. Consequently, any equilibrium profile under FIFO must have $r_{t} \leq 1$ for all $t>0$.

Secondly for part (ii): Let profile $a^{\prime}$ be a Nash equilibrium characterized by Lemma 1 , Lemma 3 part $(i)$, and $r_{0}<3$. Moreover, let $\beta=\min \left\{t \mid \sum_{t} r_{t}=3\right\}$ denote the earliest period where all players have arrived under $a^{\prime}$. Suppose that $a^{\prime}$ has $\beta=1$ such that the last player arrives at period 1 . Given the service capacity of one player per period, at least one player is served at period 2 with queuing time since period $\beta$. Lemma 3 part $(i)$, it follows that the number of arrivals at $t>0$ never exceeds the service capacity, which leaves only one possible profile with $\beta=1$; namely $[2,1]$. Given the priority of 
first arrivals under FIFO and positive queuing costs, the player arriving at period 1 could increase her expected utility by instead arriving at period 2 as she obtains lower expected queuing time for the same expected service time. Consequently, under a Nash equilibrium profile with $r_{0}<3$, the last player arrives at period 2 and is served immediately without queuing time.

The only arrival profiles that satisfies Lemma 1 and 3 are those with the arrival distribution [3], $[1,1,1]$, or $[2,0,1]$. We next examine for which relative queuing costs, $\theta$, these profiles are a Nash equilibrium.

Proposition 1. Let $a^{*}$ denote a Nash equilibrium under FIFO and $\Phi_{a^{*}}$ the corresponding equilibrium welfare. Given $\theta$, we then have the following

$\theta<1 \quad$ Profile $a^{*}$ is uniquely given by the arrival distribution [3] and provides the welfare $\Phi_{[3]}$ with $C=3$.

$\theta=1 \quad$ Multiple equilibrium profiles exist which induce the arrival distributions $[2,0,1]$ and [3]. As $[2,0,1]$ provides $C=1$, it follows that $\Phi_{[2,0,1]}>\Phi_{[3]}$.

$\theta \geq 3 \quad$ Multiple equilibrium profiles exist which induce the unique arrival distribution $[1,1,1]$ and provide the maximum welfare $\Phi_{[1,1,1]}$ with $C=0$.

There exist no pure strategy equilibrium profiles for $1<\theta<3$, thus no welfare properties are established.

Proof. We prove that $[3],[2,0,1]$, and $[1,1,1]$ are equilibrium arrival distributions under FIFO for different values of $\theta$. In what follows, let $E U\left(a_{i} \mid a\right)$ describe the expected utility for player $i$ when arriving at $a_{i}$ under profile $a$. For example, $E U(1 \mid[1,2])$ describes the expected utility for the player arriving at period 0 under the arrival distribution $[1,2]$.

$[3]$ is an equilibrium arrival distribution if: (1) $E U(0 \mid[3]) \geq E U(2 \mid[2,0,1])$ which is true for all $\theta \leq 1$; and (2) $E U(0 \mid[3]) \geq E U(1 \mid[2,1])$ which is true for all $\omega \geq 0$. Given that $\theta \leq 1$ is the binding condition for both (1) and (2), we conclude that [3] is a Nash equilibrium distribution for all $\theta \leq 1$. The arrival distribution imply that two players have to queue from period 0 to 1 , and in addition one player from period 1 to 2 . Consequently, the arrival distribution [3] has $C=3$ and provides welfare $\Phi_{[3]}$. Note that $\Phi_{[3]}$ is the minimal welfare for any possible pure strategy Nash equilibrium.

$[\mathbf{2}, \mathbf{0}, \mathbf{1}]$ is an equilibrium arrival distribution if: (1) $E U(0 \mid[2,0,1]) \geq E U(1 \mid[1,1,1])$ which is true for $\theta \leq 1$; and $(2) E U(2 \mid[2,0,1]) \geq E U(0 \mid[3])$ which is true for $\theta \geq 1$. Given that $\theta=1$ is the only binding condition for both (1) and (2), we conclude that $[2,0,1]$ is an equilibrium arrival distribution for $\theta=1$. It follows immediately that $C=1$ and thus provides welfare $\Phi_{[2,0,1]}$.

$[\mathbf{1}, \mathbf{1}, \mathbf{1}]$ is an equilibrium arrival distribution if: (1) $E U(2 \mid[1,1,1]) \geq E U(0 \mid[2,1])$ which is true for $\theta \geq 3$; and $(2) E U(1 \mid[1,1,1]) \geq E U(0 \mid[2,0,1])$ which is true for $\theta \geq 1$. Given that $\theta \geq 3$ is the binding condition, we conclude that $[1,1,1]$ is a Nash equilibrium 
profile for $\theta \geq 3$. It follows immediately that $C=0$ and thus provides the maximum welfare $\Phi_{[1,1,1]}$.

The welfare ordering across the different equilibrium arrival distributions is: $\Phi_{[3]}<$ $\Phi_{[2,0,1]}<\Phi_{[1,1,1]}$.

\section{Last-In First-Out (LIFO)}

We make some observations about a pure strategy Nash equilibrium profile under LIFO.

Lemma 4. Let $a^{*}$ be a Nash equilibrium under LIFO, then

(i) For any $t<2$ where $\sum_{t} r_{t}<3$, the subsequent period $t+1$ must have a non-zero number of arrivals independent of prior arrivals.

(ii) All players do not arrive immediately, i.e. $r_{0}<3$.

Proof. Part $(i)$ and $(i i)$ are proven separately.

First, for part $(i)$ we prove by contradiction: Let profile $a^{\prime}$ be a Nash equilibrium for which some period $t^{\prime}<2$ has $\sum_{t=0}^{t^{\prime}} r_{t}<3$. Suppose that the subsequent period $t^{\prime}+1$ has no arrivals, i.e. $r_{t^{\prime}+1}=0$. In this case, any player arriving later than $t^{\prime}+1$ could increase his expected utility by arriving instead at $t^{\prime}+1$ and be serviced immediately, since LIFO prioritizes later arrivals. Consequently, any Nash equilibrium profile under LIFO has arrivals in all subsequent periods after opening until the last player has arrived.

Secondly for part (ii): Suppose that $r_{0}=3$ such that all players arrive immediately at the opening period 0 . The probability of being served immediately at period 0 without any queuing time is then given by $\frac{1}{3}$. Accordingly, the probability of being served later than period 0 with queuing time is uniformly distributed with probability $\frac{1}{3}$ for each subsequent period. All players have the opportunity to postpone their arrival to period 0 and be served immediately without any queuing time, due to LIFO's priority of latest arrivals. Consequently, if the probability of being served immediately at period 0 is strictly lower than the aggregated probability of being served later with queuing time. Since $\frac{1}{3}<\frac{2}{3}$, it follows that the expected utility of arriving at period 0 is strictly lower than arriving at period 1. Conclusively, any Nash equilibrium profile under LIFO has $r_{0}<3$.

The only arrival profiles that satisfies Lemma 1 and 4 are those inducing the arrival distributions $[1,1,1],[1,2]$, and $[2,1]$. Similarly to FIFO, we examine for which relative queuing costs these profiles are Nash equilibrium profiles. The findings are stated in the following proposition.

Proposition 2. Let $a^{*}$ denote a Nash equilibrium under FIFO and $\Phi_{a^{*}}$ the corresponding equilibrium welfare. Given $\theta$, we then have the following

$\theta<1 \quad$ Multiple equilibrium profiles exist which induce the unique arrival distribution $[2,1]$ and provide the equilibrium welfare $\Phi_{[2,1]}$ with $C=2$.

$\theta=1 \quad$ Multiple equilibrium profiles exist which induce the arrival distributions $[1,1,1]$, $[1,2]$, or $[2,1]$. As $[1,2]$ provides $C=1$, it follows that $\Phi_{[1,1,1]}>\Phi_{[1,2]}>\Phi_{[2,1]}$. 
$\theta>1 \quad$ Multiple equilibrium profiles exist which induce the unique arrival distribution $[1,1,1]$ and provide the maximum welfare $\Phi_{[1,1,1]}$ with $C=0$.

Proof. We apply similar notation as in the proof of Proposition 1 and prove that $[1,1,1]$, $[1,2]$, and $[2,1]$ are equilibrium arrival distributions under LIFO for different values of $\theta$.

$[\mathbf{1}, \mathbf{1}, \mathbf{1}]$ is an equilibrium arrival distribution if: (1) $E U(2 \mid[1,1,1]) \geq E U(0 \mid[2,1])$ which is true for all $\theta \geq 1 ;(2) E U(1 \mid[1,1,1]) \geq E U(0 \mid[2,0,1])$ which is true for all $\theta \geq 1$; and $(3) E U(2 \mid[1,1,1]) \geq E U(1 \mid[1,2])$ which is true for all $\theta \geq 1$. Given all conditions are identical, we conclude that $[1,1,1]$ is an equilibrium arrival distribution under LIFO for all $\theta \geq 1$. The distribution provides $C=0$ and thus the maximum welfare $\Phi_{[1,1,1]}$. $[\mathbf{1 , 2}]$ is an equilibrium arrival distribution if: (1) $E U(1 \mid[1,2]) \geq E U(0 \mid[2,1])$ which is true for all $\theta \geq 1$; and $(2) E U(1 \mid[1,2]) \geq E U(2 \mid[1,1,1])$ which is true for all $\theta \leq 1$. Given that $\theta=1$ is the only binding condition for both (1) and (2), we conclude that $[1,2]$ is an equilibrium arrival distribution under LIFO for $\theta=1$. The distribution provides $C=1$ and thus welfare $\Phi_{[1,2]}$.

$[\mathbf{2 , 1}]$ is an equilibrium arrival distribution if: (1) $E U(1 \mid[2,1]) \geq E U(0 \mid[3])$ which is true for all $\delta>0$; and $(2) E U(0 \mid[2,1]) \geq E U(2 \mid[1,1,1])$ which is true for all $\theta \leq$ 1. Given that $\delta>0$ per construction, we conclude that $[2,1]$ is an equilibrium arrival distribution under LIFO for all $\theta \leq 1$. The distribution provides $C=2$ and thus welfare $\Phi_{[2,1]}$.

The welfare ordering across the different equilibrium arrival distributions is: $\Phi_{[2,1]}<$ $\Phi_{[1,2]}<\Phi_{[1,1,1]}$.

\section{Service In Random Order (SIRO)}

Unlike under FIFO and LIFO, there are no further additions to Lemma 1 for which we can exclude other arrival profiles to be Nash equilibria under SIRO. We therefore immediately turn to examining when the arrival profiles are Nash equilibrium given the relative queuing cost.

Proposition 3. Let $a^{*}$ denote a Nash equilibrium under FIFO and $\Phi_{a^{*}}$ the corresponding equilibrium welfare. Given $\theta$, we then have the following

$\theta<1 \quad$ Profile $a^{*}$ is uniquely given by the arrival distribution [3] and provides the equilibrium welfare $\Phi_{[3]}$ with $C=3$.

$\theta=1 \quad$ Multiple equilibrium profiles exist which induce the arrival distributions $[2,0,1]$, $[2,1]$, or $[3]$. As $[2,0,1]$ provides $C=1$ and $[2,1]$ provides $C=2$, it follows that $\Phi_{[2,0,1]}>\Phi_{[2,1]}>\Phi_{[3]}$.

$\theta>\frac{5}{3} \quad$ Multiple equilibrium profiles exist which induce the unique arrival distribution $[1,1,1]$ and provide the maximum welfare $\Phi_{[1,1,1]}$ with $C=0$.

For $1<\theta<\frac{5}{3}$ there exist no equilibrium profiles and therefore no welfare characteristics can be determined. 
Proof. We apply similar notation as in the proof of Proposition 1 and prove that $[1,1,1]$, $[1,2],[2,1],[2,0,1]$, and [3] are equilibrium arrival distributions under SIRO.

$[\mathbf{1}, \mathbf{1}, \mathbf{1}]$ is an equilibrium arrival distribution if: (1) $E U(2 \mid[1,1,1]) \geq E U(0 \mid[2,1])$ which is true for all $\theta \geq \frac{5}{3}$; (2) $E U(1 \mid[1,1,1]) \geq E U(0 \mid[2,0,1])$ which is true for all $\theta \geq 1$; and $(3) E U(2 \mid[1,1,1]) \geq E U(1 \mid[1,2])$ which is true for all $\theta \geq 1$. Given that $\theta \geq \frac{5}{3}$ is the binding condition for (1) through (3), we conclude that $[1,1,1]$ is an equilibrium arrival distribution under SIRO for all $\theta \geq \frac{5}{3}$ with the maximum welfare $\Phi_{[1,1,1]}$.

$[\mathbf{1}, \mathbf{2}]$ is an equilibrium arrival distribution if: (1) $E U(1 \mid[1,2]) \geq E U(0 \mid[2,1])$ which is true for all $\theta \geq 3$; and $(2) E U(1 \mid[1,2]) \geq E U(2 \mid[1,1,1])$ which is true for all $\theta \leq 1$. It follows that the conditions for (1) and (2) are conflicting and no relative queuing costs supports $[1,2]$ as an equilibrium arrival distribution under SIRO.

$[2, \mathbf{1}]$ is an equilibrium distribution if: (1) $E U(1 \mid[2,1]) \geq E U(0 \mid[3])$ which is true for all $\theta \geq 1 ;(2) E U(0 \mid[2,1]) \geq E U(2 \mid[1,1,1])$ which is true for all $\theta \leq \frac{5}{3}$; and (3) $E U(1 \mid[2,1]) \geq E U(2 \mid[2,0,1])$ which is true for all $\theta \leq 1$. Given that $\theta=1$ is the only binding condition for (1) through (3), we conclude that $[2,1]$ is an equilibrium arrival distribution under SIRO for $\theta=1$ with welfare $\Phi_{[2,1]}$.

$[\mathbf{2}, \mathbf{0}, \mathbf{1}]$ is an equilibrium distribution if: (1) $E U(2 \mid[2,0,1]) \geq E U(0 \mid[3])$ which is true for all $\theta \geq 1 ;(2) E U(0 \mid[2,0,1]) \geq E U(1 \mid[1,1,1])$ which is true for all $\theta \leq 1$; and (3) $E U(2 \mid[2,0,1]) \geq E U(1 \mid[2,1])$ which is true for all $\theta \geq 1$. Given that the only binding for (1) through (3) is $\theta=1$, we conclude that $[2,0,1]$ is an equilibrium arrival distribution under SIRO for $\theta=1$ with welfare $\Phi_{[2,0,1]}$.

[3] is an equilibrium distribution if: (1) $E U(0 \mid[3]) \geq E U(1 \mid[2,1])$ which is true for all $\theta \leq 1$; and $(2) E U(0 \mid[3]) \geq E U(2 \mid[2,0,1])$ which is true for all $\theta \leq 1$. Given identical conditions, we conclude that $[3]$ is an equilibrium arrival distribution under SIRO for all $\theta \leq 1$ with the minimum welfare $\Phi_{[3]}$.

The welfare ordering across the different equilibrium arrival distributions is: $\Phi_{[3]}<$ $\Phi_{[2,1]}<\Phi_{[2,0,1]}=\Phi_{[1,2]}<\Phi_{[1,1,1]}$.

\section{A.2 Mixed strategy equilibrium analysis}

This section presents the symmetric mixed strategy Nash equilibrium (SMNE) profiles under each classical queue discipline and establishes their corresponding equilibrium welfare for all $\theta$. We restrict the analysis only to consider SMNE in which players at most randomize over the period 0,1 , and 2 . This is motivated by the choice of experimental parameters for which only this support domain is relevant.

Let $\bar{T}=\{0,1,2\}$ denote the restricted set of arrival strategies, and moreover, let a SMNE be the list of symmetric mixed strategies $\hat{\alpha}=\left(\hat{\alpha}_{j}\right)_{j \in N}$ where $\hat{\alpha}_{i}=\hat{\alpha}_{j}$ for all $i, j \in N$. The support of a SMNE, $\hat{\alpha}$, is the set of pure strategies $a_{i} \in \bar{T}$ which are played with positive probability. Formally, we denote the support of $\hat{\alpha}$ by $S(\hat{\alpha})=\left\{a_{i}: \hat{\alpha}_{i}\left(a_{i}\right)>0\right\} \in \bar{T}$. 
In a SMNE, all players choose an identical mixed strategy profile $\hat{\alpha}_{i}$ and no player can (strictly) improve his expected utility by a unilateral arrival deviation since all potential deviations yield at most the same expected payoff.

In order to determine any SMNE, we need to compute the players' expected payoffs for all potential deviations. To do so, we define a probability vector $\mathbf{P}$ which is induced by a $\hat{\alpha}$ with support $S(\hat{\alpha})$ and probabilities $\hat{\alpha}_{i}\left(a_{i}\right), \forall a_{i} \in S(\hat{\alpha})$. The vector $\mathbf{P}$ contains the accumulated probability for all possible combinations of realized arrivals that two players can form. For example, if $\hat{\alpha}$ has the support $S(\hat{\alpha})=\{0,1\}$ then $\mathbf{P}$ contains the accumulated probability of the two players arriving either at period 0 or 1, i.e. $\mathbf{P}=\left(\hat{\alpha}_{j}(0)^{2} \hat{\alpha}_{j}(1)^{2} 2 \hat{\alpha}_{j}(0) \hat{\alpha}_{j}(1)\right)$. In other words, $\mathbf{P}$ is a vectorization of the terms generated by a binomial expansion with a number of variables equal to the cardinality of support $S(\hat{\alpha}) .{ }^{7}$ For each $\mathbf{P}$, we define a corresponding payoff vector $\mathbf{U}_{a_{i}}$ that contains the payoffs under each possible combination of arrival realizations if player $i$ arrive at $a_{i} \in S(\hat{\alpha})$. For example, if $\hat{\alpha}$ has the support $S(\hat{\alpha})=\{0,1\}$ then $\mathbf{U}_{a_{i}}=\left(E U_{i}\left[S_{i t}\left(a_{i},\{0,0\}\right)\right] \quad E U_{i}\left[S_{i t}\left(a_{i},\{1,1\}\right)\right] \quad E U_{i}\left[S_{i t}\left(a_{i},\{0,1\}\right)\right]\right)$ for each $a_{i} \in S(\hat{\alpha})$. Of course, the position of each payoff in vector $\mathbf{U}_{a_{i}}$ is matched with the position of its probability of occurrence in $\mathbf{P}$.

In a SMNE, all arrivals $a_{i} \in S(\hat{\alpha})$ must yield the same expected payoff, whereas all other arrivals outside the support domain $a_{i}^{\urcorner} \in \bar{T} \backslash\{S(\hat{\alpha})\}$ must yield at most the same payoff. We therefore have the following equilibrium conditions for any SMNE:

$$
\begin{array}{ll}
\mathbf{P U}_{a_{i}}^{\prime}=\mathbf{P U}_{a_{i}^{\prime}}^{\prime} \quad \forall a_{i}, a_{i}^{\prime} \in S(\hat{\alpha}) \\
\mathbf{P U}_{a_{i}}^{\prime} \geq \mathbf{P} \mathbf{U}_{a_{i}}^{\prime} \quad \forall a_{i} \in S(\hat{\alpha}), \forall a_{i}^{\urcorner} \in \bar{T} \backslash\{S(\hat{\alpha})\}
\end{array}
$$

To find a SMNE, we solve for $\hat{\alpha}$ the system of equations in (4) which contains a number of equations equal to the cardinality of $S(\hat{\alpha})$. To find all SMNE, we apply the following algorithm

1. Determine a support domain $S(\hat{\alpha}) \in \bar{T}$.

2. Compute $\mathbf{P}$ and $\mathbf{U}_{a_{i}}$ for all $a_{i} \in S(\hat{\alpha})$ and try to solve condition (4) for $\hat{\alpha}$ under the constraints that $0 \leq \alpha_{i}\left(a_{i}\right) \leq 1$ and $\theta \geq 0$ (note that this is done analytically).

3. Check condition (5) for any arrival $a_{i}^{\urcorner} \in \bar{T} \backslash\{S(\hat{\alpha})\}$.

4. Check that solution of $\hat{\alpha}$ is unique.

5. For the unique solution $\hat{\alpha}$, compute its values for all $\theta$.

6. Pick another support for $\hat{\alpha}$ and repeat the algorithm.

\footnotetext{
${ }^{7}$ For example, if $\hat{\alpha}$ has support $S(\hat{\alpha})=\{0,1\}$ with the set cardinality $|S(\hat{\alpha})|=2$, then the vector elements are the terms generated by the binomial expansion $\left(\hat{\alpha}_{j}(0)+\hat{\alpha}_{j}(1)\right)^{2}=\hat{\alpha}_{j}(0)^{2}+\hat{\alpha}_{j}(1)^{2}+2 \hat{\alpha}_{j}(0) \hat{\alpha}_{j}(1)$. Similarly, if if $\hat{\alpha}$ has support $S(\hat{\alpha})=\{0,1,2\}$ with the set cardinality $|S(\hat{\alpha})|=3$, then the vector elements are the terms generated by the binomial expansion $\left(\hat{\alpha}_{j}(0)+\hat{\alpha}_{j}(1)+\hat{\alpha}_{j}(2)\right)^{2}$, i.e. $\mathbf{P}=$

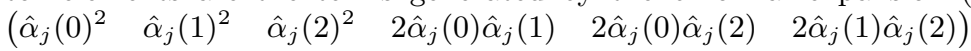


Figure 7 presents the SMNE solutions under the classical queue disciplines for every $\theta \in$ $[0,6]$. Figure $(a)$ presents the probabilities $\alpha_{i}\left(a_{i}\right)$ for each $a_{i} \in \bar{T}$, while $(b)$ presents the corresponding (indexed) equilibrium welfare. The solutions for each queue discipline are presented in separate figures. There exists a (non-degenerate) symmetric mixed strategy equilibrium for any $\theta>0$ under LIFO, while for $\theta>1$ under FIFO and SIRO due to strategic dominance of period 0 arrivals for $\theta<1$. Depending on $\theta$ and the queue discipline, the symmetric mixed strategy equilibria exists for different support sets $S(\alpha)$. Under LIFO, the mixed strategy equilibrium profile is $\alpha^{\{0,1\}}$ for $0<\theta \leq \sqrt{2}$, while $\alpha^{\{0,1,2\}}$ for $\theta>\sqrt{2}$. Under FIFO, $\alpha^{\{0,2\}}$ for $0<\theta \leq 1+2 \sqrt{2}$, while $\alpha^{\{0,1,2\}}$ for $\theta>1+2 \sqrt{2}$. Lastly under SIRO, $\alpha^{\{0,1,2\}}$ for all $\theta>1$. In terms of SMNE welfare, LIFO provides the strictly highest equilibrium welfare for any relative queuing cost, while FIFO provides the lowest. 
Figure 7: Symmetric Mixed Strategy Equilibria under Fifo, LiFO, and SiRO

(a) Mixed strategy equilibrium
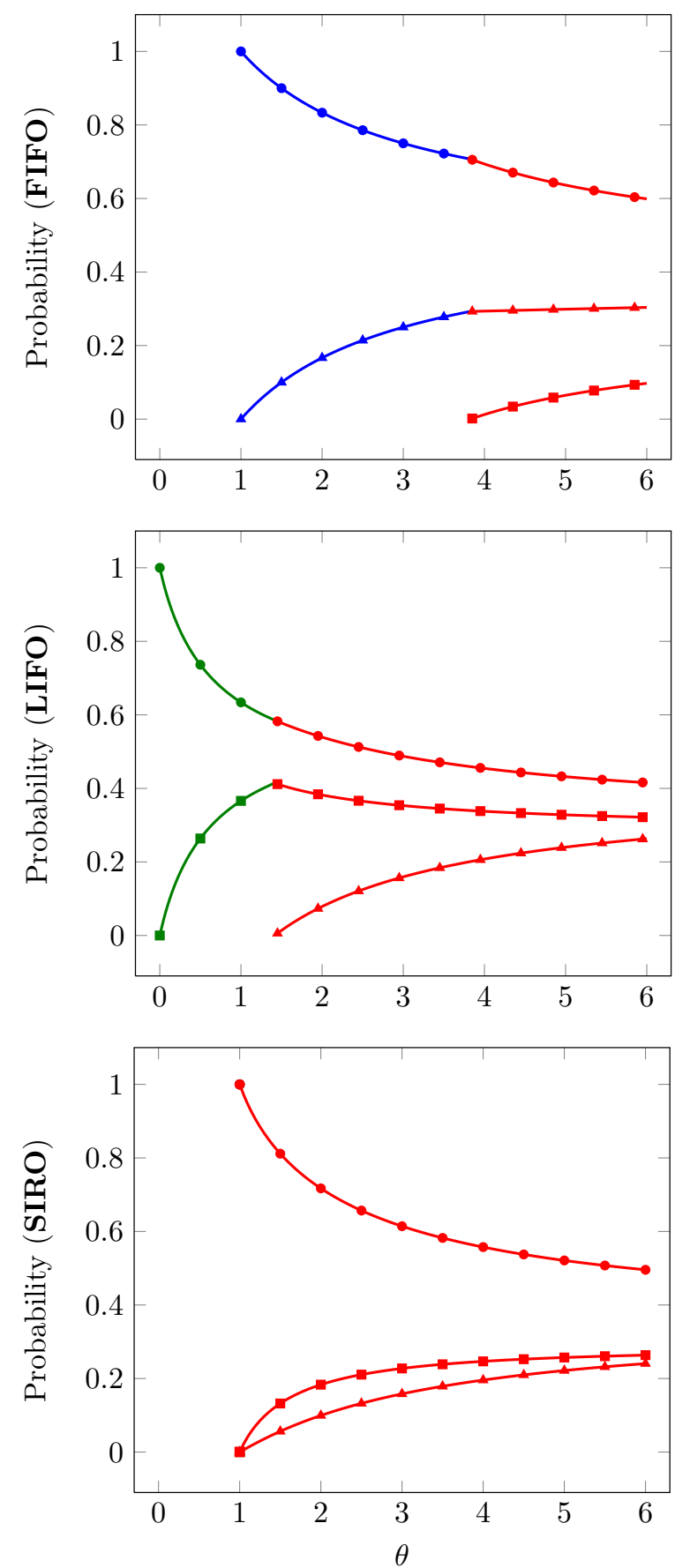

$\longrightarrow \alpha_{i}(0) \in \alpha^{\{0,1,2\}} \longrightarrow \alpha_{i}(0) \in \alpha^{\{0,2\} \longrightarrow \alpha_{i}(0) \in \alpha^{\{0,1\}}}$

$\rightarrow \alpha_{i}(1) \in \alpha^{\{0,1,2\}} \longrightarrow \alpha_{i}(2) \in \alpha^{\{0,2\} \rightarrow} \alpha_{i}(1) \in \alpha^{\{0,1\}}$

$\longrightarrow \alpha_{i}(2) \in \alpha^{\{0,1,2\}}$ (b) Expected equilibrium welfare
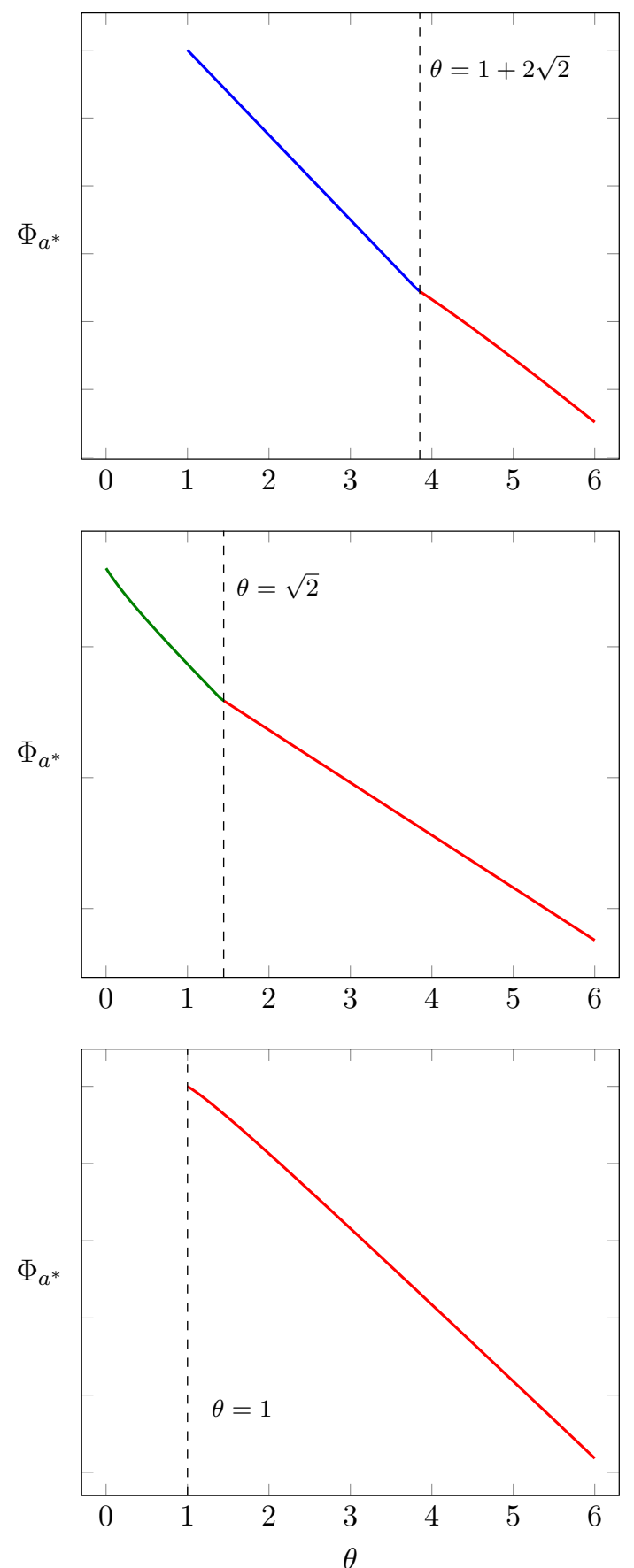

$-\Phi_{\alpha}\{0,1,2\}$

$-\Phi_{\alpha}\{0,1\}$

$-\Phi_{\alpha}\{0,2\}$ 


\section{A.3 Statistics}

Table 3: Test Statistics for Experimental Results

\begin{tabular}{|c|c|c|c|c|c|c|}
\hline \multicolumn{7}{|c|}{ Panel A: Arrival Distribution } \\
\hline \multirow[b]{2}{*}{ Disciplines } & \multicolumn{2}{|c|}{ Arrivals $\left(a_{i}\right)$} & \multicolumn{2}{|c|}{ Equilibrium difference } & \multicolumn{2}{|c|}{ Differences } \\
\hline & Mean & Std. & $H_{1}$ & $p$-value & $H_{1}$ & $p$-value \\
\hline FIFO & 0.27 & 0.58 & $F I F O>F I F O^{*}$ & $0.000^{* *}$ & $F I F O<L I F O$ & $0.000^{* *}$ \\
\hline$L I F O$ & 1.22 & 1.39 & $L I F O>L I F O^{*}$ & $0.000 * *$ & $S I R O<L I F O$ & $0.000^{* *}$ \\
\hline$S I R O$ & 0.52 & 1.13 & $S I R O>S I R O^{*}$ & $0.000 * *$ & $F I F O<S I R O$ & 0.080 \\
\hline
\end{tabular}

Panel B: Best Response Arrivals

\begin{tabular}{|c|c|c|c|c|c|c|}
\hline \multirow[b]{2}{*}{ Discipline } & \multirow[b]{2}{*}{ Share } & \multicolumn{2}{|c|}{ Best Response differences } & \multicolumn{3}{|c|}{ Arrival deviations } \\
\hline & & $H_{1}$ & $p$-value & Obs. & Mean & Std. \\
\hline FIFO & 0.79 & $F I F O>L I F O$ & $0.000^{* *}$ & 30 & 0.27 & 0.58 \\
\hline LIFO & 0.40 & $S I R O>L I F O$ & $0.000^{* *}$ & 86 & 1.22 & 1.39 \\
\hline$S I R O$ & 0.71 & $F I F O>S I R O$ & 0.101 & 41 & 0.52 & 1.13 \\
\hline
\end{tabular}

Panel C: Expected Queuing Times

\begin{tabular}{|c|c|c|c|c|}
\hline \multirow[b]{2}{*}{ Queue Discipline } & \multicolumn{2}{|c|}{ Equilibrium difference } & \multicolumn{2}{|c|}{ Differences } \\
\hline & $H_{1}$ & $p$-value & $H_{1}$ & $p$-value \\
\hline$F I F O$ & $F I F O<F I F O^{*}$ & $0.000 * *$ & $F I F O>L I F O$ & $0.000^{* *}$ \\
\hline$L I F O$ & $L I F O<L I F O^{*}$ & $0.000 * *$ & $S I R O>L I F O$ & $0.000^{* *}$ \\
\hline$S I R O$ & $S I R O<S I R O^{*}$ & $0.000 * *$ & $F I F O>S I R O$ & $0.000^{* *}$ \\
\hline
\end{tabular}

\begin{tabular}{|c|c|c|c|c|c|}
\hline \multicolumn{6}{|c|}{ Panel D: Expected PAyofF } \\
\hline \multirow{2}{*}{\multicolumn{2}{|c|}{ Queue Discipline }} & \multicolumn{2}{|c|}{ Equilibrium difference } & \multicolumn{2}{|c|}{ Differences } \\
\hline & & $H_{1}$ & $p$-value & $H_{1}$ & $p$-value \\
\hline \multicolumn{2}{|l|}{$F I F O$} & $F I F O>F I F O^{*}$ & $0.000^{* *}$ & $F I F O<L I F O$ & 0.304 \\
\hline \multicolumn{2}{|l|}{$L I F O$} & $L I F O>L I F O^{*}$ & $0.000^{* *}$ & $S I R O<L I F O$ & 0.283 \\
\hline \multicolumn{2}{|l|}{$S I R O$} & $S I R O>S I R O^{*}$ & $0.000^{* *}$ & $F I F O<S I R O$ & $0.001^{* *}$ \\
\hline \multicolumn{6}{|c|}{ Panel E: FAIRNESS } \\
\hline \multicolumn{2}{|c|}{ Ex-ante differences } & \multicolumn{2}{|c|}{ Ex-post differences } & \multicolumn{2}{|c|}{ Ex-ante vs. Ex-post } \\
\hline$H_{1}$ & $p$-value & $H_{1}$ & $p$-value & $H_{1}$ & $p$-value \\
\hline$L I F O<F I F O$ & $0.000^{* *}$ & $L I F O<F I F O$ & $0.000^{* *}$ & FIFO $O_{\text {ante }} \neq$ FIFO $O_{\text {post }}$ & 0.241 \\
\hline$L I F O<S I R O$ & $0.000 * *$ & $L I F O<S I R O$ & $0.001^{* *}$ & $L I F O_{\text {ante }} \neq L I F O_{\text {post }}$ & $0.000 * *$ \\
\hline$S I R O<F I F O$ & $0.000 * *$ & $S I R O<F I F O$ & $0.000 * *$ & $S I R O_{\text {ante }} \neq$ SIRO $O_{\text {post }}$ & 0.083 \\
\hline
\end{tabular}

Note: Significant at: ${ }^{*} 5$-percent level and ${ }^{* *} 1$-percent level. 


\section{References}

Armantier, O. and Treich, N. (2012). Eliciting beliefs: Proper scoring rules, incentive, stakes and hedging. Working Paper, Toulous School of Economics.

Arnott, R., de Palma, A., and Lindsey, R. (1993). A structural model of peak-period congestion: A traffic bottleneck with elastic demand. The American Economic Review, 83(1):161-179.

Arnott, R., de Palma, A., and Lindsey, R. (1999). Information and time-of- usage decisions in the bottleneck model with stochastic capacity and demand. European Economic Review, 43:525-548.

Avi-Itzhak, B. and Levy, H. (2004). On measuring fairness in queues. Advances in Applied Probability, 36(3):919-936.

Avi-Itzhak, B., Levy, H., and Raz, D. (2011). Principles of Fairness Quantification in Queueing Systems. In Network Performance Engineering: Lecture Notes in Computer Sciences, 5233, Kouvatsos D (ed.). Springer-Verlag.

Bell, D. E. (1985). Disappointment in decision making under uncertainty. Operations Research, 33(1):1-27.

Camerer, C. F. (2003). Behavioral game theory: Experiments in strategic interaction. Princeton University Press.

Conlisk, J. (1996). Why bounded rationality? Journal of Economic Literature, 34:669-700.

de Palma, A. and Fosgerau, M. (2011). Dynamic Traffic modeling, in A de Palma, R Lindsey, E. Quinet and R. Vickerman (eds), A Handbook of Transport Economics. Edward Elgar Publishing, Inc.

de Palma, A. and Fosgerau, M. (2013). Random queues and risk averse users. European Journal of Operational Research, 230(2):313-320.

Ellsberg, D. (1961). Risk, ambiguity, and the savage axioms. Quarterly Journal of Economics, 75:643-669.

Fehr, E. and Schmidt, K. M. (1999). A theory of fairness, competition, and cooperation. Quarterly Journal of Economics, 114(3):817-868.

Fischbacher, U. (2007). z-tree: Zurich toolbox for ready-made economic experiments. Experimental Economics, 10(2):171-178.

Fudenberg, D. and Levine, D. K. (1998). The Theory of Learning in Games. MIT Press.

Glazer, A. and Hassin, R. (1983). ?/M/1: On the equilibrium distribution of customer arrivals. European Journal of Operational Research, 13(2):146-150.

Grether, D. M. and Plott, C. R. (1979). Economic theory of choice and the preference reversal phenomenon. American Economic Review, 69(4):623-38.

Hall, R. W. (1991). Queueing methods: for services and manufacturing, volume 1. Prentice Hall Englewood Cliffs, NJ.

Hassin, R. (1985). On the optimality of first come last served queues. Econometrica, 53(1):201-02.

Hassin, R. and Haviv, M. (2003). To Queue or not to Queue: Equilibrium Behavior in Queueing Systems. Springer.

Hassin, R. and Kleiner, Y. (2011). Equilibrium and optimal arrival patterns to a server with opening and closing times. IIE Transactions, 43:164-175.

Hillier, F. S. (1990). Introduction To Operations Research. Tata McGraw-Hill Education, 8th edition.

Jain, R., Juneja, S., and Shimkin, N. (2011). The concert queueing problem: to wait or to be late. Discrete Event Dynamic Systems, 21(1):103-138.

Juneja, S. and Shimkin, N. (2013). The concert queueing game: Strategic arrivals with waiting and tardiness costs. Queueing Systems, 74(4):369-402.

Kingman, J. F. C. (1962). The effect of queue discipline on waiting time variance. Mathematical Proceedings of the Cambridge Philosophical Society, 58(1):163-164.

Norman, G. (2010). Likert scales, levels of measurement and the "laws" of statistics. Advances in Health Science Education, 15(5):625-632.

Ostubo, H. and Rapoport, A. (2008). Vickrey's model of traffic congestion discretized. Transportation Research Part B: Methodological, 42:873-889. 
Palfrey, T. R. and Wang, S. W. (2009). On eliciting beliefs in strategic games. Journal of Economic Behavior and Organization, 71(2):98-109.

Platz, T. T. and Østerdal, L. P. (2012). The curse of the first-in-first-out queue discipline. Discussion Papers of Business and Economics, University of Southern Denmark, No. 10/2012.

Rafaeli, A., Barron, G., and Haber, K. (2002). The effects of queue structure on attitudes. Journal of Service Research, 5(2):125-139.

Rafaeli, A., Kedmi, E., Vashdi, D., and Barron, G. (2005). Queues and fairness: A multiple study investigation. Technical report, Technion, Israel Institute of Technology, Haifa, Israel.

Rapoport, A., Stein, W. E., Parco, J. E., and Seale, D. A. (2004). Equilibrium play in single-server queues with endogenously determined arrival times. Journal of Economic Behavior and Organization, 55(1):67-91.

Seale, D. A., Parco, J. E., Stein, W. E., and Rapoport, A. (2005). Joining a queue or staying out: Effects of information structure and service time on arrival and staying out decisions. Experimental Economics, $8(2): 117-144$.

Small, K. A. and Verhoef, E. T. (2007). The Economics of Urban Transportation. Routledge, New York.

Tversky, A. and Kahnemann, D. (1974). Judgment under uncertainty: Heuristics and biases. Science, 185(4157):1124-1131.

Vickrey, W. S. (1969). Congestion theory and transport investment. The American Economic Review, $59(2): 251-60$. 